\title{
Atomic-Scale Tuning of Graphene/Cubic SiC Schottky Junction for Stable Low-Bias Photoelectrochemical Solar-to-Fuel Conversion
}

\author{
Hao Li, Yuchen Shi, Huan Shang, Weimin Wang, Jun Lu, Alexei A. Zakharov, Lars Hultman, \\ Roger I. G. Uhrberg, Mikael Syväjärvi, Rositsa Yakimova, Lizhi Zhang, and Jianwu Sun*
}

Cite This: ACS Nano 2020, 14, 4905-4915

Read Online

ACCESS | Lلll Metrics \& More | 回 Article Recommendations | st Supporting Information

ABSTRACT: Engineering tunable graphene-semiconductor interfaces while simultaneously preserving the superior properties of graphene is critical to graphene-based devices for electronic, optoelectronic, biomedical, and photoelectrochemical applications. Here, we demonstrate this challenge can be surmounted by constructing an interesting atomic Schottky junction via epitaxial growth of high-quality and uniform graphene on cubic $\mathrm{SiC}$ (3C-SiC). By tailoring the graphene layers, the junction structure described herein exhibits an atomic-scale tunable Schottky junction with an inherent built-in electric field, making it a perfect prototype to systematically comprehend interfacial electronic properties and transport mechanisms. As a proof-of-concept study, the atomicscale-tuned Schottky junction is demonstrated to promote both the separation and transport of charge carriers in a typical photoelectrochemical system for solar-to-fuel conversion under low bias. Simultaneously, the as-grown monolayer graphene with an extremely high conductivity protects the surface

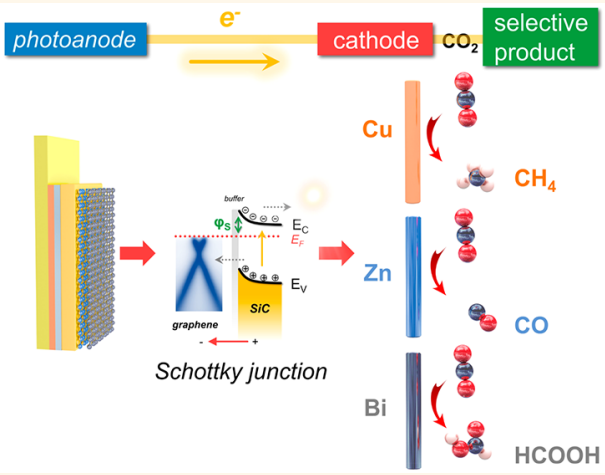
of $3 \mathrm{C}-\mathrm{SiC}$ from photocorrosion and energetically delivers charge carriers to the loaded cocatalyst, achieving a synergetic enhancement of the catalytic stability and efficiency.

KEYWORDS: SiC, graphene, Schottky junction, photoelectrochemistry, $\mathrm{CO}_{2}$ reduction

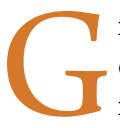
raphene, a single layer of graphite with closely packed conjugated hexagonal lattices, is one of the thinnest materials in the universe. Due to its unique geometric and electronic structures, graphene possesses many intriguing properties, including excellent conductivity, high transparency, great stability, flexibility, and elasticity. These exciting properties endow graphene great potential for applications in electronic, optoelectronic, biomedical, and photoelectrochemical areas. ${ }^{1,2}$ Unfortunately, free-standing graphene is rather limited for these applications. For instance, the lack of intrinsic band gap (zero band gap) impedes its implementation into conventional electronics due to substantial leakage currents in field-effect devices and also makes it a poor material for solar light harvesting or hot carrier generation. ${ }^{3-6}$ In light of this fact, the large-scale application of graphene is usually associated with the integration of graphene into current semiconductor technology. ${ }^{7}$ One of the key factors that determines the performances of graphene-based devices is the quality of the graphene-semiconductor interface, where a
Schottky junction is usually formed. Apart from quality, the tunability of such a Schottky junction is also of particular importance to control and gain mechanistic insight into interfacial electronic properties and transport mechanisms for applications in photodetectors, sensors, and solar cells. Besides quality and tunability, another aspect that matters a lot over the graphene-semiconductor junction is the electronic structure of integrated graphene, which, in many cases, is extremely sensitive to its bonding structure and chemical environment. This concern can be exemplified by the unique $100 \% \mathrm{sp}^{2}$-hybridized states of graphene, which will be broken if graphene is strongly bonded to adjacent substrates,

Received: February 4, 2020

Accepted: April 3, 2020

Published: April 3, 2020 
a

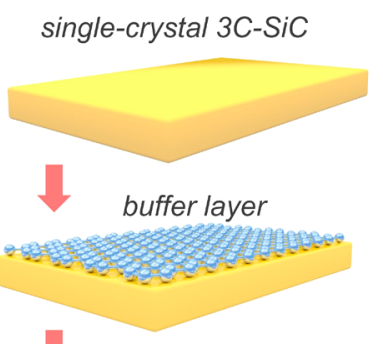

monolayer graphene

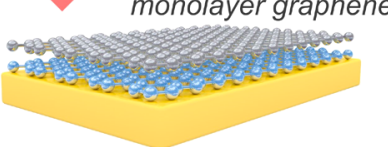

bilayer graphene

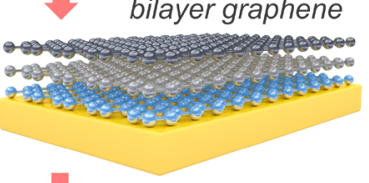

4-layer graphene

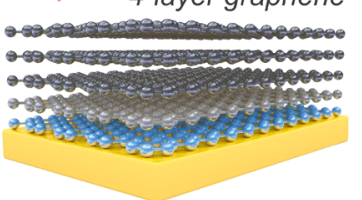

b
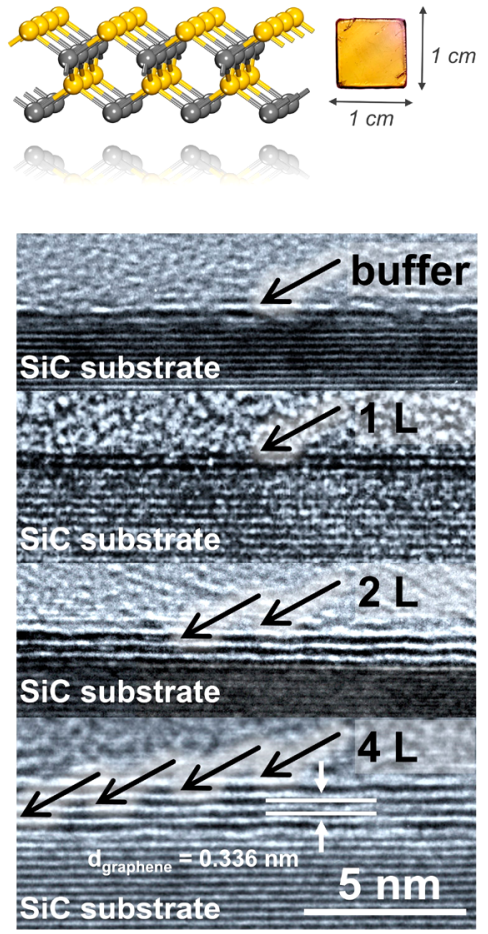

d

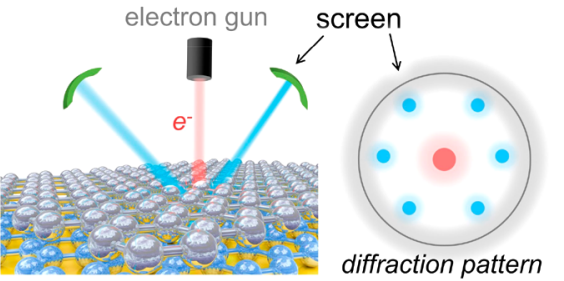

e

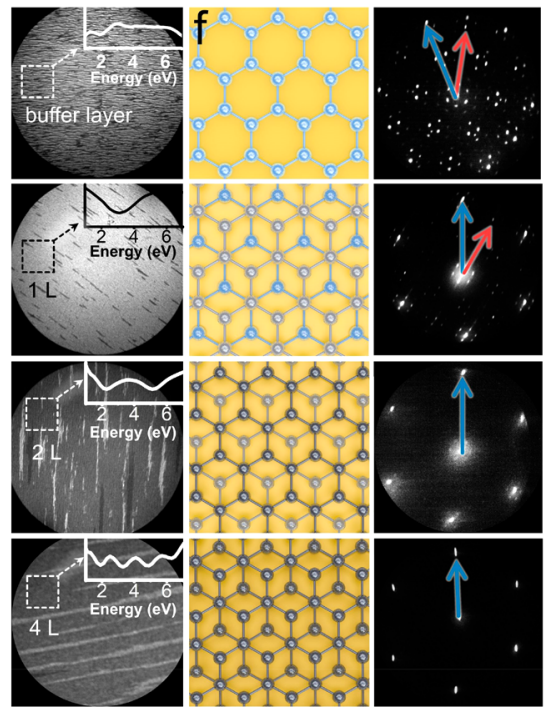

Figure 1. Synthesis and characterization of graphene/3C-SiC samples. (a) Schematic illustration of the preparation process. (b) Optical image and crystal structure of the yellowish single-crystal 3C-SiC substrate. (c) Cross-sectional HRTEM images of the buffer layer, monolayer (1L), bilayer (2L), and four-layer (4L) graphene samples grown on 3C-SiC. (d) Schematic illustration of low-energy electron diffraction (LEED) measurements. (e) Low-energy electron microscopy (field of view $=5 \mu \mathrm{m}$ ) and (f) LEED images of the as-grown graphene/3C-SiC samples. Two reciprocal unit vectors are presented in the LEED patterns, which indicate one each for 3C-SiC (red/short) and graphene (blue/long). The insets in (e) show the electron reflectivity curves collected from the labeled regions. The number of graphene layers is determined by the number of dips in the electron reflectivity curves.

thus disturbing the $\pi$ electrons and lowering the intrinsic inplane conductivity. ${ }^{8}$ Particularly, for graphene layers that are produced through chemical exfoliation of graphite or chemical vapor deposition on metals, their superior properties are deteriorated during the required transfer process to semiconductor substrates, often with residues of processing chemicals. Therefore, establishing a highly qualified tunable graphene-semiconductor junction interface, while simultaneously preserving the superior properties of graphene without chemical-structural modification, is the key challenge for the engineering of graphene-based devices.

Multilayer graphene, consisting of two or more layers of graphene, is of particular interest due to its unique interlayer interaction and tunable electronic band structure. ${ }^{9,10}$ This is because the addition of each individual graphene layer will modify the integral electronic structure and induce charge transfer density modulations at the interface, thus producing a different material with peculiar properties. ${ }^{8,11,12}$ Herein, we report the construction of an atomic-scale tunable graphene/ 3C-SiC Schottky junction by tailoring the number of graphene layers via an epitaxial growth technique. High-quality and uniform graphene can be in situ grown on 3C-SiC, and the number of graphene layers can be precisely controlled, thus avoiding a conventional graphene transfer process. Meanwhile, the superior properties of graphene, including the high conductivity, transparency, and stability, are well-preserved along with the graphene growth. Most importantly, the formation of an atomic Schottky junction at the graphene/
3C-SiC interface, with a tunable barrier and the built-in electric field, enables us to systematically control and comprehend the interfacial electronic properties and transport mechanisms of graphene-based devices. Benefiting from these merits, the atomically sharp graphene/3C-SiC Schottky junction is demonstrated to tackle three great challenges of photoelectrochemical catalysis: the limited light absorption, the undesirable charge recombination, and instability of photoelectrodes. ${ }^{13-15}$ As a proof-of-concept study, the junction structure described herein also exhibits a synergetic enhancement of both the stability and the efficiency of photoelectrochemical solar-to-fuel conversion.

\section{RESULTS AND DISCUSSION}

The epitaxial growth of graphene layers on 3C-SiC was performed by the thermal decomposition (sublimation) process of $3 \mathrm{C}-\mathrm{SiC}(111)$ substrates (Figure 1a,b and Figure S1, Supporting Information). ${ }^{16}$ A considerable advantage of this technique is that graphene can be in situ produced, without etchant chemicals, on a large area with extremely high quality and uniformity, thus ensuring the high quality of the graphene-semiconductor junction structure. No additional transfer process is required to fabricate electronic devices as graphene is directly grown on the $3 \mathrm{C}-\mathrm{SiC}$ semiconductor in a controlled manner. Through adjusting the growth temperature and time, large-area monolayer (1L), bilayer (2L), and fourlayer (4L) graphene were demonstrated to be readily in situ grown on $3 \mathrm{C}-\mathrm{SiC}$ substrates in this work. As revealed by cross- 
a

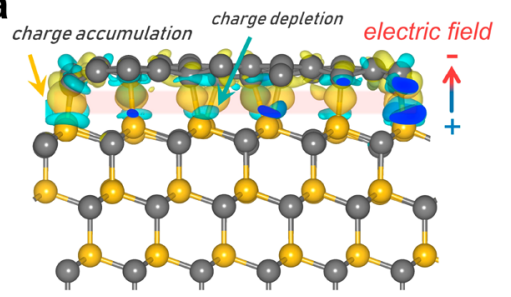

e
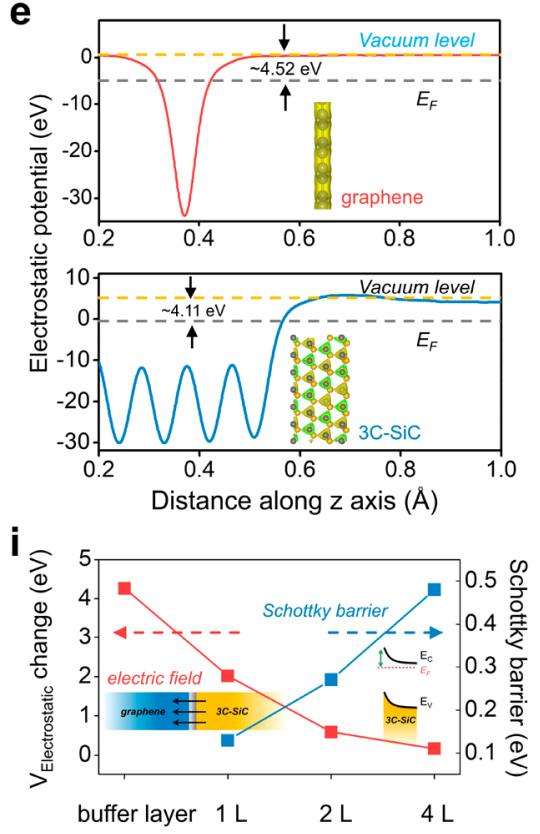

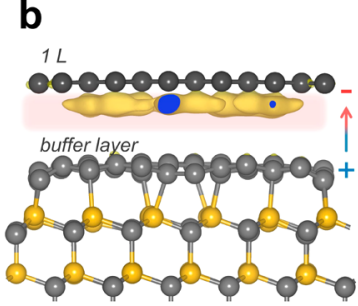

$f$

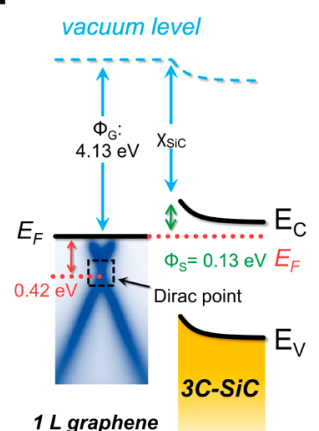

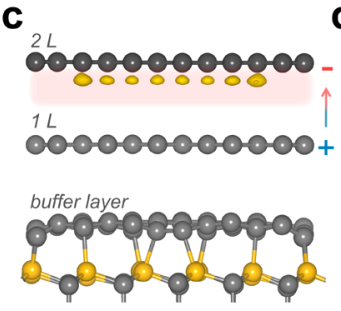

g

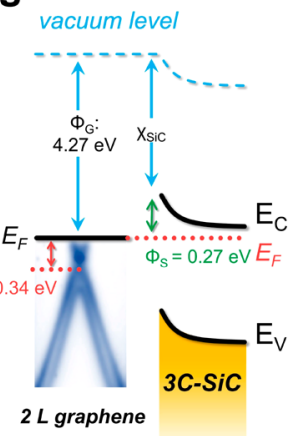

d

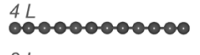

$3 \mathrm{~L}$ $3 L 00000000000$ $2 L$ 年0000000000 $1 L 0000000000$ buffer layer opopoposog

h

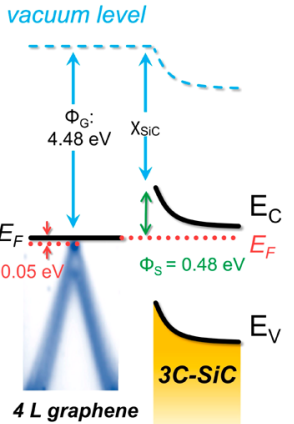

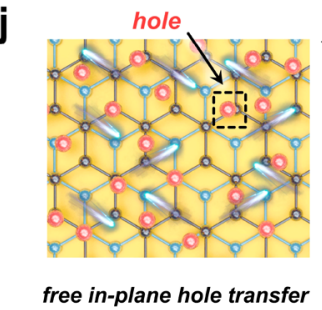
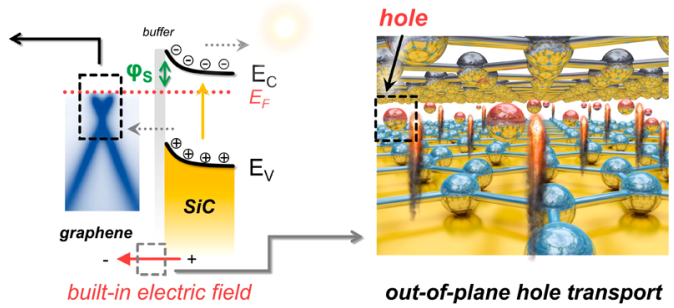

Figure 2. Electronic structure of graphene/3C-SiC samples. (a) Charge density difference for buffer layer/3C-SiC. Yellow and blue isosurfaces $(0.004 \mathrm{au})$ represent charge accumulation and depletion in the space with respect to the isolated buffer layer and $3 \mathrm{C}-\mathrm{SiC}$. Charge density difference between the two outermost atomic layers of (b) $1 \mathrm{~L} / 3 \mathrm{C}-\mathrm{SiC},(\mathrm{c}) 2 \mathrm{~L} / 3 \mathrm{C}$-SiC, and (d) $4 \mathrm{~L} / 3 \mathrm{C}$-SiC. The yellow isosurface represents charge accumulation in the spatial graphene region in the presence of the $3 \mathrm{C}-\mathrm{SiC}$ substrate. (e) Electrostatic potential diagrams of graphene and the 3C-SiC surface obtained from DFT calculations. Energy band structures for (f) $1 \mathrm{~L} / 3 \mathrm{C}-\mathrm{SiC},(\mathrm{g}) 2 \mathrm{~L} / 3 \mathrm{C}-\mathrm{SiC}$, and $(\mathrm{h}) 4 \mathrm{~L} /$ 3C-SiC. (i) Schottky barrier height of the graphene/3C-SiC junction and the difference of electrostatic potential energy $\left(V_{\text {Electrostatic }}\right)$ between the two outermost atomic graphene as a function of the total number of graphene layers. (j) Schematic illustration of the excitation and charge transport process under solar light irradiation.

sectional observation using high-resolution transmission electron microscope (HRTEM), growth of graphene on 3C$\mathrm{SiC}$ was first associated with the formation of a buffer layer, which is constituted of carbon atoms with a honeycomb structure and is strongly bonded to $\mathrm{Si}$ atoms of the $3 \mathrm{C}-\mathrm{SiC}$ surface (Figure 1c). ${ }^{17-19}$ By adjusting the growth condition (see Methods), the $1 \mathrm{~L}, 2 \mathrm{~L}$, and $4 \mathrm{~L}$ graphene layers can be controllably grown on top of the buffer layer of 3C-SiC (Figure 1c). Our previous work has demonstrated that $4 \mathrm{~L}$ graphene shows an $\mathrm{ABCA}$ (rhombohedral) stacking sequence. ${ }^{20}$ It is reported that $3 \mathrm{C}-\mathrm{SiC}(111)$ with the $\mathrm{ABC}$ stacking along the [111] direction is favorable for producing rhombohedralstacked multilayer graphene. ${ }^{11}$ Note that all graphene samples presented here contain the buffer layer, which is commonly observed in graphene samples grown on $\mathrm{Si}$-face $\mathrm{SiC}$ substrates and serves as the precursor for the subsequent growth of monolayer and multilayer graphene. However, the buffer layer does not possess typical electronic properties of graphene due to the strong chemical bonding. ${ }^{21,22}$ The cross-sectional HRTEM image shows that the graphene interlayer spacing is around $0.336 \mathrm{~nm}$ in the $4 \mathrm{~L}$ graphene, close to the reported spacing of multilayer graphene.

Low-energy electron microscopy (LEEM) was then employed to specifically characterize the uniformity and the number of graphene layers grown on 3C-SiC. Together with LEEM, low-energy electron diffraction (LEED) could further collect the information on the buffer layer, the quality of graphene layers, as well as the detailed surface structure of these single-crystalline materials according to the diffraction spots of electrons (Figure 1d). According to LEEM images, the buffer layer, the monolayer, and multilayer graphene were controllably grown on 3C-SiC (Figure 1e). Corresponding graphene layers were determined by the number of dips in electron reflectivity curves shown in the insets of Figure 1e. ${ }^{24}$ For $3 \mathrm{C}-\mathrm{SiC}$ with a buffer layer (BL/3C-SiC), monolayer graphene $(1 \mathrm{~L} / 3 \mathrm{C}-\mathrm{SiC})$, bilayer graphene $(2 \mathrm{~L} / 3 \mathrm{C}-\mathrm{SiC})$, and four-layer graphene $(4 \mathrm{~L} / 3 \mathrm{C}-\mathrm{SiC})$, the coverage ratio is 80,91 , 87 , and $70 \%$, respectively. The LEED results confirmed the presence of $(6 \sqrt{ } 3 \times 6 \sqrt{ } 3) R 30^{\circ}$ diffraction spots of the buffer layer, which did not exhibit any graphene electronic properties due to a covalent chemical bonding between the buffer layer and $3 \mathrm{C}-\mathrm{SiC}{ }^{16,25}$ Along with graphene growth, sharp and bright $(1 \times 1)$ graphene diffraction spots gradually dominated the LEED patterns for $1 \mathrm{~L}, 2 \mathrm{~L}$, and $4 \mathrm{~L}$ graphene samples, confirming a high quality of the as-grown crystalline graphene (Figure 1e). The Raman results corroborated that the buffer layer and high-quality graphene with different layers had been 
successfully grown on 3C-SiC substrates (Figure S2, Supporting Information).

Successful growth of high-quality graphene layers on 3C-SiC allowed us to systematically study their interfacial and electronic structures. Density functional theory (DFT) was first adopted to investigate interfacial charge exchange before and after the contact between $3 \mathrm{C}-\mathrm{SiC}$ and graphene layers. Buffer layer was found to be partially bonded with $\mathrm{Si}$ atoms of the $3 \mathrm{C}-\mathrm{SiC}$ substrate via part of its $\pi$ electrons, which explained why the buffer layer was not electronically active and could not be considered as graphene (Figure 2a). ${ }^{22}$ Meanwhile, the charge density difference calculation revealed a redistribution of charge density, as reflected by a significant electron flow from $3 \mathrm{C}-\mathrm{SiC}$ to the buffer layer that led to a formation of a built-in electric field (Figure 2a). ${ }^{4,26}$ The charge transfer modulations induced by the buffer layer at the interface was evidenced by X-ray photoelectron spectroscopy depth profiling analysis (Figure S3, Supporting Information). In addition to the prominent geometric interaction, the buffer layer was found to electronically couple with $3 \mathrm{C}-\mathrm{SiC}$ according to its density of states (DOS) that is strongly hybridized with that of $3 \mathrm{C}-\mathrm{SiC}$ within the band gap (Figure S4a, Supporting Information). These strong interactions between $3 \mathrm{C}-\mathrm{SiC}$ and the buffer layer arising from their atomically sharp discontinuity are beneficial for rapid thermal equilibration, as well as charge transfer. ${ }^{7,27}$ Interestingly, DFT calculations further show that the built-in electric field formed between the buffer layer and $3 \mathrm{C}-\mathrm{SiC}$ can be further extended into the outermost graphene layer in both $1 \mathrm{~L} / 3 \mathrm{C}-\mathrm{SiC}$ and $2 \mathrm{~L} / 3 \mathrm{C}-\mathrm{SiC}$ (Figure $2 \mathrm{~b}, \mathrm{c}$ and Figure S4b, Supporting Information). This is due to the further donation of electrons from the $3 \mathrm{C}-\mathrm{SiC} /$ buffer layer interface to the outermost graphene layer. However, without the buffer layer, interfacial exchange between $3 \mathrm{C}-\mathrm{SiC}$ and graphene layers would be barely possible. This unambiguously indicates that the trapping of electrons by the buffer layer is irreplaceable for the overall charge transfer from $3 \mathrm{C}-\mathrm{SiC}$ to graphene layers to reach the thermal equilibrium condition. ${ }^{28}$ When the number of graphene layers was increased to four, the polarization effect and corresponding built-in electric field between the two outermost graphene layers became extremely weak, indicating a negligible charge transfer to the fourth graphene layer (Figure 2d). ${ }^{29}$ Thus, DFT calculations clearly show that the buffer layer with a short-range effect is critical to mediate electron transfer to the first and second but not to the fourth graphene layer.

The electron exchange between $3 \mathrm{C}-\mathrm{SiC}$ and graphene would result in the formation of an atomic Schottky junction with a built-in electric field between $3 \mathrm{C}-\mathrm{SiC}$ and graphene layers. ${ }^{30}$ To prove this point, we calculated the work functions of $3 \mathrm{C}$ $\mathrm{SiC}$ and free-standing graphene through DFT. According to the potential lineups and the distance between the vacuum level and the Fermi level, free-standing graphene displays an average work function of $4.52 \mathrm{eV}$ (Figure 2e), much higher than that of the $3 \mathrm{C}-\mathrm{SiC}(111)$ surface $(4.11 \mathrm{eV})$. An energy band diagram for free-standing graphene and $3 \mathrm{C}-\mathrm{SiC}$ prior to thermal equilibrium was proposed, where $E_{\mathrm{F}}$ was the Fermi energy, $E_{\mathrm{C}}$ the conduction band edge, and $E_{\mathrm{V}}$ the valence band edge (Figure S5a, Supporting Information). Theoretically, due to the work function of $3 \mathrm{C}-\mathrm{SiC}$ being lower than that of graphene, electrons are expected to flow from $3 \mathrm{C}-\mathrm{SiC}$ to graphene until their Fermi levels are aligned at equilibrium upon contact, leading to the formation of a Schottky junction with a built-in electric field directed from $3 \mathrm{C}-\mathrm{SiC}$ to graphene, as obtained by our DFT calculations (Figure S5b, Supporting Information).

To check our interpretation of a Schottky junction formation, angle-resolved photoelectron spectroscopy (ARPES) was then employed to measure the energy band structure and Fermi levels of graphene samples. For the freestanding graphene, the $E_{\mathrm{F}}$ of graphene is expected to be located to its Dirac point $\left(E_{\mathrm{D}}\right)$. Due to electron transfer to graphene, it gave rise to a high electron concentration in the graphene and lifted the $E_{\mathrm{F}}$ above the Dirac point. ${ }^{20,30,31}$ As seen in Figure $2 \mathrm{f}, \mathrm{g}$, the ARPES results reveal that the $E_{\mathrm{F}}$ is located above the Dirac point. Relative to the Dirac point, an energy difference $\left(E_{\mathrm{F}}-E_{\mathrm{D}}\right)$ of 0.42 and $0.34 \mathrm{eV}$ was observed for $1 \mathrm{~L}$ and $2 \mathrm{~L}$ graphene, respectively. For the $4 \mathrm{~L}$ graphene, $E_{\mathrm{F}}$ is almost at the Dirac point with an $E_{\mathrm{F}}-E_{\mathrm{D}}$ value falling into the ARPES resolution limit of $0.05 \mathrm{eV}$ (Figure $2 \mathrm{~h}$ ). The decreased $E_{\mathrm{F}}-E_{\mathrm{D}}$ value of the $4 \mathrm{~L}$ graphene is consistent with the drastically decreased electron transfer to the fourth graphene layer according to the DFT calculation, which reflects a much lowered charge carrier concentration on the $4 \mathrm{~L}$ graphene. Therefore, by increasing the number of graphene layers, the electron concentration in outer-layer graphene is decreasing and $E_{\mathrm{F}}$ is gradually shifted toward to the Dirac point, which indicates an increased work function. Meanwhile, the width of the flat band at $E_{\mathrm{F}}$ is measured to be $\sim 0.07 \AA^{-1}$, which is quite close to the calculated value of the flat band region of $0.06 \AA^{-1}$ for rhombohedral $4 \mathrm{~L}$ graphene with a $\mathrm{ABCA}$ stacking sequence (Figure S6, Supporting Information). ${ }^{20,32}$

To determine the work function of the as-grown graphene $\left(\Phi_{\mathrm{G}}\right)$ of different layers, ultraviolet photoemission spectroscopy (UPS) was employed. According to the UPS results shown in Figure S7a, the work functions of $1 \mathrm{~L}, 2 \mathrm{~L}$, and $4 \mathrm{~L}$ graphene were calculated to be $4.13,4.27$, and $4.48 \mathrm{eV}$, respectively (Figure $2 \mathrm{f}-\mathrm{h}$ ). Thus, the work function of graphene increases with increasing the number of the graphene layers. Notably, the work function $(4.48 \mathrm{eV})$ of $4 \mathrm{~L}$ graphene is very close to the intrinsic value $(4.52 \mathrm{eV})$ of free-standing graphene, consistent with the ARPES result showing that its $E_{\mathrm{F}}$ is almost located at the Dirac point (Figure $2 \mathrm{~h}$ and Figure S7b, Supporting Information). This can be explained by the following. As shown by the DFT calculations above, the buffer layer plays a key role in transferring electrons to the first and second graphene layer but not to the fourth graphene layer. The transferred charges should be distributed among the graphene layers with a reduced charge density toward the outer layers, while a constant Fermi level should be kept for the whole system. The fourth graphene layer is in an almost neutral state due to the negligible charge transfer from its underneath layers, which is the very reason why its Fermi level is close to the intrinsic Fermi level at the Dirac point. To quantify the Schottky junction, we calculated the Schottky barrier height $\left(\varphi_{\mathrm{S}}\right)$ by the equation of $\Phi_{\mathrm{S}}=\Phi_{\mathrm{G}}-\chi_{\mathrm{SiC}}$, in which $\chi_{\mathrm{SiC}}$ is the electron affinity of $3 \mathrm{C}-\mathrm{SiC}\left(\chi_{\mathrm{SiC}}=4.00 \mathrm{eV}\right) .^{33}$ Based on the measured work functions, the Schottky barrier heights of the $1 \mathrm{~L} / 3 \mathrm{C}-\mathrm{SiC}, 2 \mathrm{~L} / 3 \mathrm{C}-\mathrm{SiC}$, and $4 \mathrm{~L} / 3 \mathrm{C}-\mathrm{SiC}$ junctions were calculated to be $0.13,0.27$, and $0.48 \mathrm{eV}$ (Figure 2f-h), respectively. This demonstrates that the Schottky barrier height increased with the increase of stacking graphene layers.

As mentioned above, another important parameter of the Schottky junction is the built-in electric field, $\overrightarrow{E_{i}}$. We define the absolute value of $\overrightarrow{E_{i}}$ between the two outermost atomic layers 


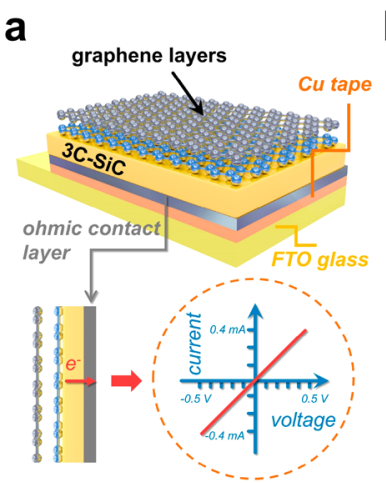

b

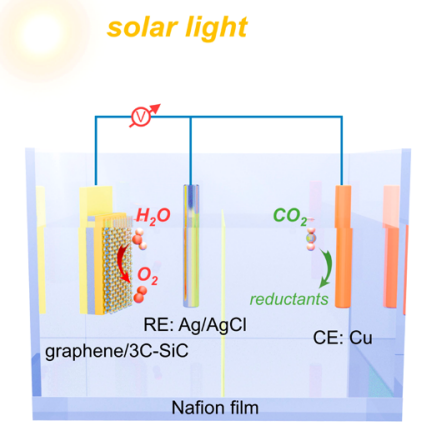

d

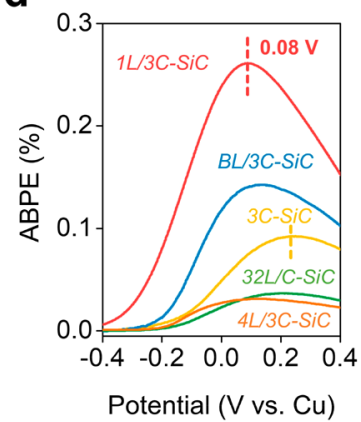

e

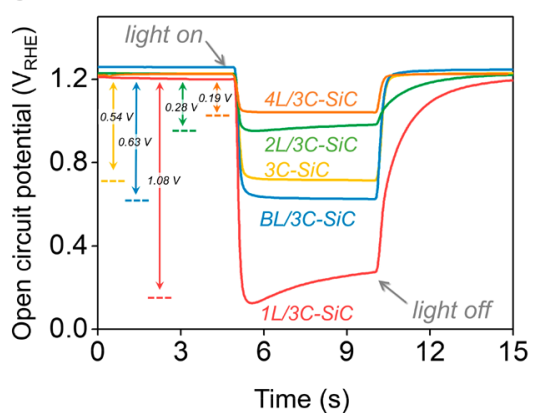

C

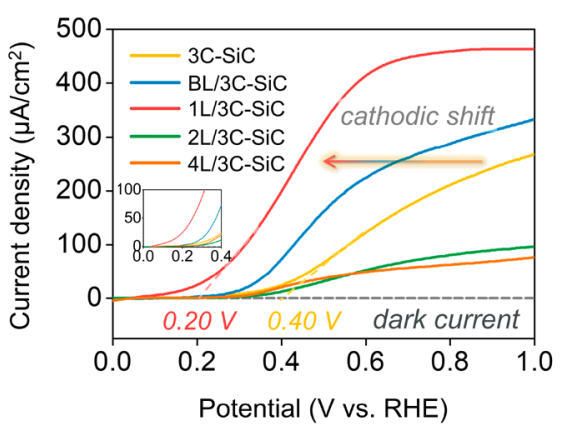

f

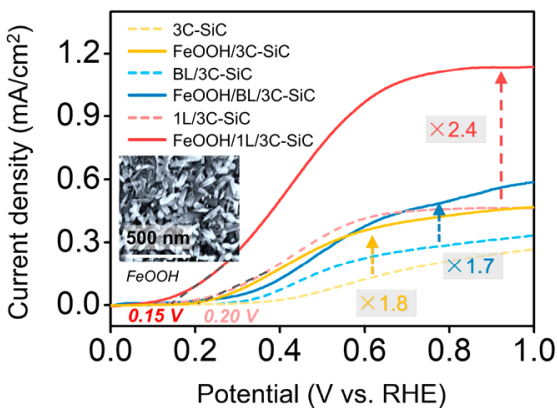

Figure 3. Photoelectrochemical performance of the graphene/3C-SiC photoanodes. (a) Schematic illustration of the photoanode structure and a representative I-V curve of the Ohmic contact. (b) Schematic illustration of a two-compartment PEC cell separated by the Nafion membrane for water oxidation and $\mathrm{CO}_{2}$ reduction. (c) Current density-voltage $(J-V)$ curves of the graphene/3C-SiC photoanodes in the PEC cell under illumination and (d) corresponding applied bias photon-to-current conversion efficiency. (e) Open-circuit potentials of the graphene/3C-SiC photoanodes in the dark and light. (f) $J-V$ curves of the graphene/3C-SiC photoanodes loaded with FeOOH cocatalyst under illumination. Inset shows the scanning electron microscopy image of the FeOOH nanorods. Light source: simulated solar light (AM1.5G, $100 \mathrm{~mW} / \mathrm{cm}^{2}$ ). Electrolyte: $0.5 \mathrm{M} \mathrm{pH} \mathrm{7.5} \mathrm{KHCO}_{3}$ solution. Counter electrode: $\mathrm{Cu}$.

by the equation $E_{i}=\left|\frac{1}{e} \nabla V_{i}\right|$, in which $V_{i}$ is the energy difference of the electrostatic potential between the two outermost atomic layers. According to the calculated electrostatic potential energy diagram, the value of $V_{i}$ between $3 \mathrm{C}-\mathrm{SiC}$ and the buffer layer reached the maximum due to their strong chemical interaction (Figure 2i and Figure S8a, Supporting Information). The value of $V_{i}$ between buffer layer and $1 \mathrm{~L}$ graphene decreased to $2.02 \mathrm{eV}$, and the value further decreased to $0.59 \mathrm{eV}$ for $V_{i}$ between $1 \mathrm{~L}$ and $2 \mathrm{~L}$ graphene (Figure S8b,c, Supporting Information). For $4 \mathrm{~L} / 3 \mathrm{C}-\mathrm{SiC}$, the energy difference of electrostatic potential $V_{i}$ between the third and fourth graphene layers was only $0.17 \mathrm{eV}$ (Figure S8d, Supporting Information), which was negligible compared with the $V_{i}$ between buffer layer and $1 \mathrm{~L}$ graphene. The weakened builtin electric field along with multilayer graphene stacking could also be reflected in the cross-sectional HRTEM image of $4 \mathrm{~L} /$ 3C-SiC. Between the buffer layer and $1 \mathrm{~L}$ graphene, a slight expansion of the lattice spacing up to $0.362 \mathrm{~nm}$ is observed due to the strong interaction between the buffer layer and the 3C$\mathrm{SiC}$ substrate (Figure S9, Supporting Information). However, above the $1 \mathrm{~L}$ graphene, interlayer lattice spacing $(0.336 \mathrm{~nm})$ is close to the theoretical value $(0.340 \mathrm{~nm})$, which is consistent with the weak interfacial built-in electric field. As a result, along with multilayer graphene growth, the built-in electric field between graphene layers continuously decreased until it almost vanished at the fourth graphene layer, whereas the Schottky barrier height of the graphene/3C-SiC junctions gradually increased (Figure 2i).
In principle, the graphene/3C-SiC structures with a tunable Schottky junction and electric field will allow us to systematically understand the carrier separation and interfacial charge transport behaviors, both of which are critical to the efficiency of photoelectrochemical (PEC) cells for solar-to-fuel conversion. This assumption is first supported by the small band gap $\left(E_{\mathrm{g}}=2.36 \mathrm{eV}\right)$ of $3 \mathrm{C}-\mathrm{SiC}$ for sufficient visible sunlight absorption in comparison with that of $6 \mathrm{H}-\mathrm{SiC}\left(E_{\mathrm{g}}=3.03 \mathrm{eV}\right)$ and $4 \mathrm{H}-\mathrm{SiC}\left(E_{\mathrm{g}}=3.26 \mathrm{eV}\right)$. Also, $3 \mathrm{C}-\mathrm{SiC}$ possesses suitable energy band positions that straddle the redox potential of water splitting (Figure S10, Supporting Information). Meanwhile, high transparency ( $\sim 97 \%)$ of graphene ensures the transmission of almost all solar light, and high stability of graphene may also serve as a protective material to prevent the $\mathrm{SiC}$ surface from corrosion or destruction. Suppose that 3C$\mathrm{SiC}$ is excited by solar light, electrons will be excited to the $E_{\mathrm{C}}$, leaving holes in the $E_{\mathrm{V}}$. The photogenerated holes can be readily swept into graphene, whereas electrons are injected toward the backside of $3 \mathrm{C}-\mathrm{SiC}$ by the built-in electric field (Figure 2j). For holes in graphene, their in-plane mobility can be considered as barrierless due to the high conductivity of graphene. Meanwhile, the directional built-in electric field may also enable an accelerated out-of-plane transport route for photogenerated holes, which is usually considered as sluggish due to the van der Waals forces between graphene layers (Figure $2 \mathrm{j}$ ). The out-of-plane transport of photogenerated holes is of considerable importance as holes are apt to diffuse out of graphene for a surface chemical reaction. It is also to be noted that the in situ growth of graphene layers without any transfer process preserved the geometric and electronic 
structures of graphene, including its high conductivity, and the high conductivity of graphene in our case can potentially act as an ideal platform to enable reliable cocatalyst loading for more efficient interfacial charge transfer.

To test our hypothesis, we used the as-grown graphene/3C$\mathrm{SiC}$ samples to construct photoanodes by directly depositing ohmic contacts. A $300 \mathrm{~nm}$ thick $\mathrm{Al}$ was deposited on the backside of the single-crystal 3C-SiC, and a layer of $\mathrm{Au}(200$ $\mathrm{nm}) / \mathrm{Ti}(5 \mathrm{~nm})$ was deposited on the backside of graphene/ $3 \mathrm{C}-\mathrm{SiC}$ samples. Both of them form ohmic contact, showing a linear $I-V$ curve (Figure $3 \mathrm{a}$ and Figure S11, Supporting Information).$^{34}$ In a two-compartment PEC cell separated by the Nafion membrane, the photoanodes can be used for water oxidation in combination with $\mathrm{CO}_{2}$ on a commercial metal cathode ( $\mathrm{Cu}$ cathode) for the production of solar fuels (Figure 3b). ${ }^{35} \mathrm{~A} 0.5 \mathrm{M} \mathrm{pH} 7.5 \mathrm{KHCO}_{3}$ solution was used as the electrolyte in our case. The anode compartment was continuously bubbled with $\mathrm{Ar}$ gas, while the cathode compartment was bubbled with $\mathrm{CO}_{2}$ of high purity. The PEC water oxidation on the as-prepared photoanodes was first evaluated, and the photoanodes showed a negligible dark current in the linear sweep voltammetry scan. Under simulated 1 sun irradiation (AM1.5G, $\left.100 \mathrm{~mW} / \mathrm{cm}^{2}\right), 3 \mathrm{C}-\mathrm{SiC}$ showed an onset potential of around $0.20 \mathrm{~V}$ versus the reversible hydrogen electrode $\left(\mathrm{V}_{\mathrm{RHE}}\right)$ and a sluggish photocurrent increase around $0.40 \mathrm{~V}_{\mathrm{RHE}}$ in the $J-V$ curve (Figure $3 \mathrm{c}$ ). After the growth of the buffer layer, $\mathrm{BL} / 3 \mathrm{C}-\mathrm{SiC}$ showed an onset potential similar to that of $3 \mathrm{C}-\mathrm{SiC}$ with a slightly increased photocurrent (Figure 3c). As the buffer layer strongly interacted with 3C-SiC through chemical bonds, it is electronically inactive in terms of the high conductivity of graphene, which could explain why the BL/3C-SiC photoanode only showed a slightly increased photocurrent. $^{22}$ Interestingly, the $1 \mathrm{~L} / 3 \mathrm{C}-\mathrm{SiC}$ photoanode exhibited an almost zero onset potential of water oxidation, and a precipitous photocurrent increase occurred at as low as $0.20 \mathrm{~V}_{\mathrm{RHE}}$, a $0.20 \mathrm{~V}$ cathodic shift relative to the $3 \mathrm{C}-\mathrm{SiC}$ or $\mathrm{BL} / 3 \mathrm{C}-\mathrm{SiC}$ photoanode (Figure 3c). Meanwhile, the saturated photocurrent density around $450 \mu \mathrm{A} / \mathrm{cm}^{2}$ with a plateau appeared as low as $0.60 \mathrm{~V}_{\mathrm{RHE}}$ for the $1 \mathrm{~L} / 3 \mathrm{C}$-SiC photoanode. However, further increase of the graphene layers to $2 \mathrm{~L}$ and $4 \mathrm{~L}$ resulted in a photocurrent drop with increased onset potential (Figure 3c). According to the ARPES results and measured work functions, 2L/3C-SiC and $4 \mathrm{~L} / 3 \mathrm{C}-\mathrm{SiC}$ possessed a Schottky barrier height higher than that of $1 \mathrm{~L} / 3 \mathrm{C}-\mathrm{SiC}$, which would theoretically facilitate the carrier separation. However, the aforementioned calculations demonstrated that the further increase of the graphene layers resulted in a decreased electric field between the outermost graphene layers. This decreased electric field would hinder the out-of-plane hole transport across the multiple graphene layer, which could explain the drop in photocurrent response for the $2 \mathrm{~L} / 3 \mathrm{C}-\mathrm{SiC}$ and $4 \mathrm{~L} / 3 \mathrm{C}-\mathrm{SiC}$ photoanodes.

The reduced onset potential of the $1 \mathrm{~L} / 3 \mathrm{C}-\mathrm{SiC}$ photoanode suggested a reduced overpotential for water oxidation, as further evidenced by applied bias photon-to-current efficiency (ABPE) (Figure 3d). The maximum ABPE around $0.26 \%$ was achieved at an extremely low bias of $0.08 \mathrm{~V}$ for the $1 \mathrm{~L} / 3 \mathrm{C}-\mathrm{SiC}$ photoanode under simulated solar light, whereas it required a higher applied bias of $0.21 \mathrm{~V}$ to reach a maximum ABPE of $0.1 \%$ for the $3 \mathrm{C}-\mathrm{SiC}$ photoanode. The increased photocurrent response of $1 \mathrm{~L} / 3 \mathrm{C}-\mathrm{SiC}$ was consistent with its highest photovoltage $(1.08 \mathrm{~V})$, almost 2 times that of $3 \mathrm{C}-\mathrm{SiC}(0.54$ $\mathrm{V}$ ) (Figure $3 \mathrm{e}$ ). Further increase of the graphene layers to $2 \mathrm{~L}$ and $4 \mathrm{~L}$ resulted in a decreased photovoltage, agreeing well with the lowered photocurrent. To observe the shift of the flat band potential of 3C-SiC, we measured the Mott-Schottky plots of the as-prepared graphene/3C-SiC junctions at a frequency of 1 $\mathrm{kHz}$. For $3 \mathrm{C}-\mathrm{SiC}$, the positive slope is consistent with that of typical n-type semiconductors, and according to the intercept of the linear region, the flat band potential is around -0.20 $\mathrm{V}_{\mathrm{RHE}}$ (Figure S12a, Supporting Information). When the buffer layer was incorporated, the flat band potential of $3 \mathrm{C}-\mathrm{SiC}$ was slightly shifted from -0.20 to $-0.21 \mathrm{~V}_{\mathrm{RHE}}$. Interestingly, the incorporation of $1 \mathrm{~L}, 2 \mathrm{~L}$, and $4 \mathrm{~L}$ graphene on $3 \mathrm{C}-\mathrm{SiC}$ showed an identical and more negatively shifted flat band potential at $-0.24 \mathrm{~V}_{\mathrm{RHE}}$ (Figure S12a, Supporting Information). The Mott-Schottky plots display comparable slopes due to the identical doping concentration around $7.5 \times 10^{15} \mathrm{~cm}^{-3}$ in $3 \mathrm{C}$ $\mathrm{SiC}$. The identical flat band potential of $1 \mathrm{~L} / 3 \mathrm{C}-\mathrm{SiC}, 2 \mathrm{~L} / 3 \mathrm{C}-$ $\mathrm{SiC}$, and $4 \mathrm{~L} / \mathrm{SiC}$ suggests that this negative flat band shift is possibly due to the passivation of surface defects by graphene layers. ${ }^{36}$ Some surface states cause a potential drop within the Helmholtz layer that can be minimized through surface passivation. ${ }^{37}$

We also measured the flat band potential of $3 \mathrm{C}-\mathrm{SiC}$ at a higher frequency. The flat band potential measuring a higher frequency of $5 \mathrm{kHz}$ was estimated to be $-0.21 \mathrm{~V}_{\mathrm{RHE}}$, close to that measured under $1 \mathrm{kHz}$. Moreover, a similar cathodic shift of the flat band potential was also observed after the incorporation of $1 \mathrm{~L}$ graphene (Figure S12b, Supporting Information). In comparison with $3 \mathrm{C}-\mathrm{SiC}$ and $\mathrm{BL} / 3 \mathrm{C}-\mathrm{SiC}$, the higher flat band potential of $1 \mathrm{~L} / 3 \mathrm{C}-\mathrm{SiC}$ is consistent with the tendency of its higher photovoltage. However, based on the flat potential alone, we cannot explain the higher photovoltage of $1 \mathrm{~L} / 3 \mathrm{C}-\mathrm{SiC}$ and the lowered photovoltage of $2 \mathrm{~L} / 3 \mathrm{C}-\mathrm{SiC}$ and $4 \mathrm{~L} / 3 \mathrm{C}-\mathrm{SiC}$. This, on the other hand, indicates that the large photovoltage generated by $1 \mathrm{~L} / 3 \mathrm{C}-\mathrm{SiC}$ not only is due to more negative flat band potential but also is closely related to the desirable built-in electric field. It is worth noting that, for the open-circuit potential (OCP) measurements, $\mathrm{O}_{2}$ gas was continuously bubbled into the electrolyte $(0.5 \mathrm{M} \mathrm{pH}$ $7.5 \mathrm{KHCO}_{3}$ ), and each dark/light OCP was obtained with constant stirring. As expected, the dark OCP values are close to $\sim 1.23 \mathrm{~V}_{\mathrm{RHE}}$. Under light excitation, the photogenerated carriers result in the photovoltage under the open-circuit condition. For the $1 \mathrm{~L} / 3 \mathrm{C}-\mathrm{SiC}$, the monolayer graphene not only suppressed the surface recombination by passivating the surface defects of 3C-SiC but also formed an optimal Schottky junction at the interface of $1 \mathrm{~L} / 3 \mathrm{C}-\mathrm{SiC}$, thus enhancing the separation of photogenerated carriers. Therefore, the $1 \mathrm{~L} / 3 \mathrm{C}$ $\mathrm{SiC}$ photoanode delivered the highest photovoltage. For $3 \mathrm{C}$ $\mathrm{SiC}$ photoanodes with thicker graphene layers, the reduced interlayer electric field hinders the out-of-plane hole transport toward the electrolyte, thus giving rise to a lower photovoltage.

To study the origin of the enhanced PEC water oxidation performance of $1 \mathrm{~L} / 3 \mathrm{C}-\mathrm{SiC}$, the electrochemical impedance spectroscopy (EIS) was used to investigate charge transfer resistance. The Nyquist plots of 3C-SiC and graphene/3C-SiC junctions were fitted using the equivalent circuits, as shown in the inset of Figure S13. Under light excitation, the equivalent circuit consists of series resistance $\left(R_{\mathrm{s}}\right)$, charge transfer resistance from the bulk to the photoanode surface $\left(R_{\mathrm{ct}}\right)$, and the charge transfer resistance from the photoanode to the electrolyte $\left(R_{\mathrm{ct} \text {,trap }}\right)$. The value of $R_{\mathrm{ct}}$ decreased from 177 to $115 \Omega \mathrm{cm}^{2}$ when $3 \mathrm{C}-\mathrm{SiC}$ was grown with $1 \mathrm{~L}$ graphene, which demonstrates that the $1 \mathrm{~L} / 3 \mathrm{C}-\mathrm{SiC}$ Schottky junction reduced 

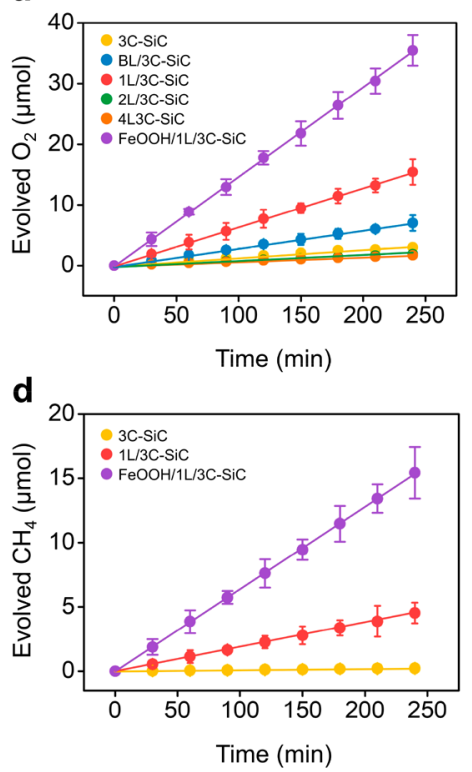
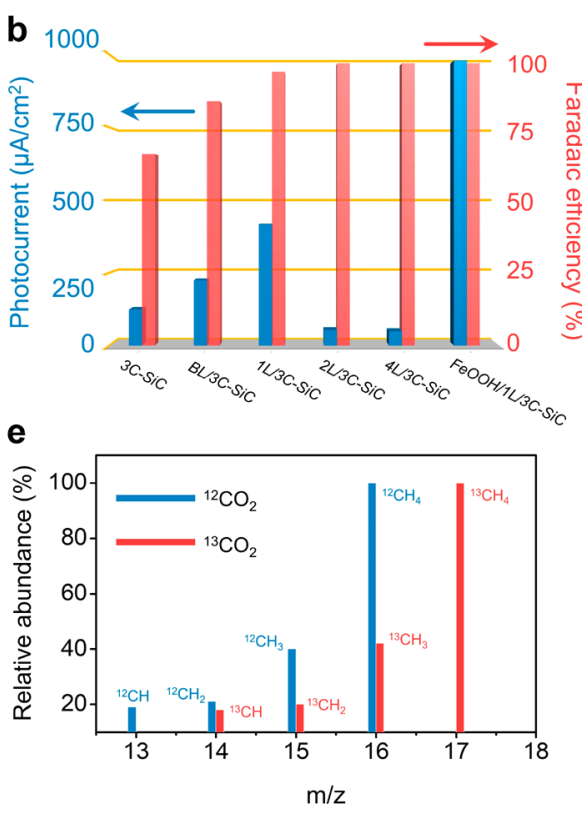

C

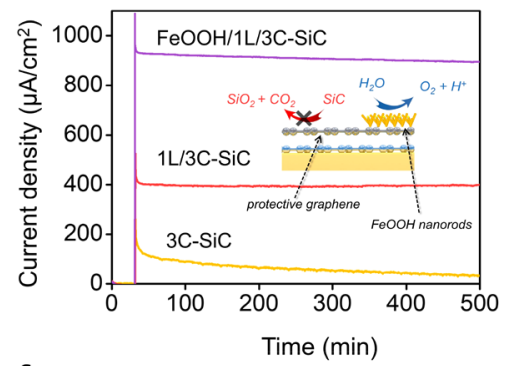

$\mathbf{f}$

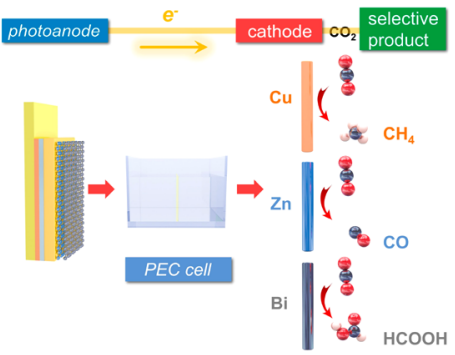

Figure 4. Photoelectrochemical $\mathrm{CO}_{2}$ reduction in combination with water oxidation on the graphene/3C-SiC photoanodes. (a) Detected $\mathrm{O}_{2}$ production over the photoanodes at a bias of $0.6 \mathrm{~V}_{\mathrm{RHE}} \cdot(\mathrm{b})$ Steady-state photocurrent and faradaic efficiency for water oxidation over the photoanodes at a bias of $0.6 \mathrm{~V}_{\mathrm{RHE}}$ (c) Comparison of the $J-t$ behavior of the $3 \mathrm{C}-\mathrm{SiC}, 1 \mathrm{~L} / 3 \mathrm{C}-\mathrm{SiC}$, and $\mathrm{FeOOH}-\mathrm{coated} 1 \mathrm{~L} / 3 \mathrm{C}-\mathrm{SiC}$ photoanodes at a bias of $0.6 \mathrm{~V}_{\mathrm{RHE}}$. (d) Generation of $\mathrm{CH}_{4}$ on $\mathrm{Cu}$ cathode. (e) Gas chromatograms of $\mathrm{CH}_{4}$ evolved from isotopic ${ }^{13} \mathrm{CO}_{2}$ as carbon source using $\mathrm{FeOOH} / 1 \mathrm{~L} / 3 \mathrm{C}$-SiC as the photoanode and $\mathrm{Cu}$ as the cathode (results using ${ }^{12} \mathrm{CO}_{2}$ as carbon source are given as a comparison). (f) Schematic illustration of the proof-of-concept PEC cell for a selective conversion of $\mathrm{CO}_{2}$ into solar fuels using the FeOOH/ 1L/3C-SiC photoanode. Light source: simulated solar light (AM1.5G, $100 \mathrm{~mW} / \mathrm{cm}^{2}$ ). Electrolyte: $0.5 \mathrm{M} \mathrm{pH} \mathrm{7.5} \mathrm{KHCO}_{3}$ solution. Error bars represent standard deviation based on five independent experiments.

the resistance for charge transfer from $3 \mathrm{C}-\mathrm{SiC}$ to $1 \mathrm{~L}$ graphene (Table S1, Supporting Information). Meanwhile, $R_{\mathrm{ct}, \text { trap }}$ of $1 \mathrm{~L} /$ 3C-SiC $\left(649 \Omega \mathrm{cm}^{2}\right)$ was also decreased compared with that of 3C-SiC (1719 $\left.\Omega \mathrm{cm}^{2}\right)$, suggesting a much smaller charge transfer resistance from the $1 \mathrm{~L} / 3 \mathrm{C}-\mathrm{SiC}$ photoanode to electrolyte for water oxidation. For $2 \mathrm{~L} / 3 \mathrm{C}-\mathrm{SiC}$ and $4 \mathrm{~L} / 3 \mathrm{C}$ $\mathrm{SiC}$, their much larger $R_{\mathrm{ct}}$ and $R_{\mathrm{ct} \text { trap }}$ values were consistent with the difficult charge transfer ability across the multiple graphene layers, as we demonstrated above (Table S1, Supporting Information).

Therefore, it was concluded that the formation of an atomic Schottky junction between graphene and 3C-SiC played an indispensable role in improving water oxidation efficiency by accelerating charge separation. However, the addition of graphene layers would reduce the out-of-plane charge transport toward an electrolyte for water oxidation due to the decreased built-in electric field between the graphene layers. Thus, tunability of the atomic Schottky junction enables a trade-off balance between Schottky barrier height and the electric field distributed across graphene layers, which can be balanced and optimized to simultaneously enhance electron-hole separation and the carrier transport across graphene layers. As the asprepared $3 \mathrm{C}-\mathrm{SiC}$ in our study is n-type conductive, a complementary evaluation of the hole transport properties (particularly in the out-of-plane direction across the graphene layers) through Hall effect measurements will be obtained when the semi-insulating $3 \mathrm{C}$-SiC substrate can be prepared in the future, which is one of our research goals for the design of other $2 \mathrm{D} /$ semiconductor heterostructures.

As we discussed above, the preserved ultrahigh in-plane conductivity of graphene could act as an ideal platform to enable efficient charge transfer into the loaded cocatalysts for an improved PEC efficiency. This notion is demonstrated by the deposition of the traditional oxygen evolution cocatalyst, FeOOH. Through a facile hydrothermal method, vertical $\mathrm{FeOOH}$ nanorods with a thickness around $200 \mathrm{~nm}$ could be deposited on the $1 \mathrm{~L} / 3 \mathrm{C}$-SiC photoanode according to the scanning electron microscopy and TEM images (Figure S14ac, Supporting Information). Combined X-ray diffraction (XRD), TEM, and X-ray photoelectron spectroscopy (XPS) studies revealed the deposition of as-grown $\mathrm{FeOOH}$ nanorods on the 1L/3C-SiC photoanode (Figure S14d-f, Supporting Information). According to the $J-V$ curve, loading the layer of $\mathrm{FeOOH}$ nanorods on pristine $3 \mathrm{C}-\mathrm{SiC}$ or $\mathrm{BL} / 3 \mathrm{C}-\mathrm{SiC}$ photoanode $(\mathrm{FeOOH} / \mathrm{BL} / 3 \mathrm{C}-\mathrm{SiC})$ with a buffer layer did not significantly improve the photocurrent density (Figure 3f). This is probably due to the sluggish hole transfer from the electronically inactive buffer layer to $\mathrm{FeOOH}$. For the $1 \mathrm{~L} / 3 \mathrm{C}$ $\mathrm{SiC}$ with a layer of highly conductive monolayer graphene, loading of $\mathrm{FeOOH}(\mathrm{FeOOH} / 1 \mathrm{~L} / 3 \mathrm{C}-\mathrm{SiC})$ could result in an over 1-fold increase of the photocurrent up to $1.13 \mathrm{~mA} / \mathrm{cm}^{2}$ (Figure 3f). According to the Mott-Schottky plots, the loading of $\mathrm{FeOOH}$ caused only a slight cathodic shift of the flat band potential of $1 \mathrm{~L} / 3 \mathrm{C}-\mathrm{SiC}$ (from -0.24 to $-0.25 \mathrm{~V}_{\mathrm{RHE}}$ ), suggesting that the electronic structure, including the Schottky barrier at the junction, was not influenced by $\mathrm{FeOOH}$ loading (Figure S12c, Supporting Information). Therefore, the $\mathrm{FeOOH}$ mainly functioned as an efficient oxygen evolution reaction cocatalyst to enhance the kinetics of water oxidation on the $1 \mathrm{~L} / 3 \mathrm{C}-\mathrm{SiC}$ photoanode according to the sharper photocurrent increase at relatively lower applied potential (Figure $3 \mathrm{f}$ ). As further revealed by fitted impedance arc radius of $\mathrm{FeOOH} / 1 \mathrm{~L} / 3 \mathrm{C}-\mathrm{SiC}, R_{\mathrm{ct}}$ and $R_{\mathrm{ct} \text {,trap }}$ values decreased to 51 and $179 \Omega \mathrm{cm}^{2}$, respectively (Table S1, Supporting Information). This suggests $\mathrm{FeOOH}$ on the conductive graphene layer not only facilitated bulk charge carrier 
separation but also decreased the charge transfer barrier for water oxidation at the electrode interface. Using $\mathrm{CoOOH}$ as another oxygen evolution reaction cocatalyst, we further demonstrated that the PEC performance of 3C-SiC could be "universally" improved with the incorporation of the graphene interlayer (Figure S15, Supporting Information). It has to be pointed out that the maximum photocurrent density of $3 \mathrm{C}-\mathrm{SiC}$ with cocatalysts is still much lower than the theoretical value calculated from its band gap. This is mainly related to the fact that the indirect band gap of $3 \mathrm{C}-\mathrm{SiC}$ results in a much larger light penetration depth than the sum of the width of space charge region $(W)$ and the carrier diffusion length $\left(L_{\mathrm{D}}\right)$. Consequently, most photogenerated carriers are distributed in a neutral region and recombine there, and only a small part of photogenerated carriers within the region of $\left(W+L_{\mathrm{D}}\right)$ can contribute to the photocurrent (see details in Figure S10d, Supporting Information). Moreover, our previous work has reported the presence of defects such as stacking faults and double positioning boundaries in the $3 \mathrm{C}-\mathrm{SiC}$ bulk that can act as bulk charge recombination centers. ${ }^{38}$

Online $\mathrm{O}_{2}$ detection via gas chromatography (GC) revealed that the generated photocurrent on $3 \mathrm{C}-\mathrm{SiC}$ was not solely associated with $\mathrm{O}_{2}$ production under a bias of $0.6 \mathrm{~V}_{\mathrm{RHE}}$ due to self-oxidation by holes $\left(3 \mathrm{C}-\mathrm{SiC}+4 \mathrm{H}_{2} \mathrm{O}+8 \mathrm{~h}^{+} \rightarrow \mathrm{SiO}_{2}+\mathrm{CO}_{2}\right.$ $+8 \mathrm{H}^{+}$) (Figure 4a,b). ${ }^{39,40}$ Interestingly, the growth of buffer layer and $1 \mathrm{~L}$ graphene not only enhanced the water oxidation efficiency of $3 \mathrm{C}-\mathrm{SiC}$ but also improved the faradaic efficiency of the photoanode from $72 \%$ (3C-SiC) to $96 \%$ (1L/3C-SiC), as shown in Figure $4 a, b$. The enhanced faradaic efficiency and the improved stability of $1 \mathrm{~L} / 3 \mathrm{C}-\mathrm{SiC}$ according to the longterm $J-t$ curve were due to the antioxidation behavior of graphene that prevented the $3 \mathrm{C}-\mathrm{SiC}$ photoanode from photocorrosion (Figure 4c). ${ }^{40}$ For the FeOOH-loaded 1L/ 3C-SiC photoanode, stability could still be maintained (Figure $4 \mathrm{c})$. As expected from the fact that graphene could protect $3 \mathrm{C}$ $\mathrm{SiC}$ from photocorrosion, the $2 \mathrm{~L} / 3 \mathrm{C}-\mathrm{SiC}$ and $4 \mathrm{~L} / 3 \mathrm{C}-\mathrm{SiC}$ with multilayer graphene also displayed good stability ( $\sim 98 \%$ faradaic efficiency). However, the amount of generated $\mathrm{O}_{2}$ on the $2 \mathrm{~L} / 3 \mathrm{C}-\mathrm{SiC}$ and $4 \mathrm{~L} / 3 \mathrm{C}-\mathrm{SiC}$ photoanodes was low due to their poor photocurrent response (Figure $4 \mathrm{~b}$ ).

In the PEC system with $3 \mathrm{C}-\mathrm{SiC}$ as the photoanode and $\mathrm{Cu}$ as the cathode, the reduction products in the cathode compartment under a saturated $\mathrm{CO}_{2}$ atmosphere were predominantly $\mathrm{H}_{2}(63.0 \%)$ with $\mathrm{CH}_{4}(13.6 \%)$ as the additional product under a bias of $0.6 \mathrm{~V}_{\mathrm{RHE}}$ (Table S2, Supporting Information). Interestingly, when $1 \mathrm{~L} / 3 \mathrm{C}-\mathrm{SiC}$ was used as the photoanode, $\mathrm{CH}_{4}(60.5 \%)$ became as the dominant product under the same bias. Selectivity of $\mathrm{CO}_{2}$ reduction to $\mathrm{CH}_{4}$ could be further increased to $82.8 \%$ when FeOOH-loaded 1L/ $3 \mathrm{C}-\mathrm{SiC}$ was used as the photoanode (Table S2, Supporting Information). The faradaic efficiency of $82.8 \%$ for $\mathrm{CH}_{4}$ in the present work is the highest ever in photoelectrochemical $\mathrm{CO}_{2}$ reduction in comparison with previous works on $\mathrm{CO}_{2}$ reduction with $\mathrm{CH}_{4}$ as the product and among the highest in the literature for electrochemical $\mathrm{CO}_{2}$ reduction (Table S3, Supporting Information). The observed high selectivity for the rapid $\mathrm{CH}_{4}$ formation in our PEC system indicated that multiple-electron $\mathrm{CO}_{2}$ reduction process $\left(\mathrm{CO}_{2}+8 \mathrm{H}^{+}+8 e^{-} \rightarrow\right.$ $\left.\mathrm{CH}_{4}+2 \mathrm{H}_{2} \mathrm{O}\right)$ would be favored using the $1 \mathrm{~L} / 3 \mathrm{C}-\mathrm{SiC}$ and $\mathrm{FeOOH} / 1 \mathrm{~L} / 3 \mathrm{C}-\mathrm{SiC}$ photoanodes that could achieve large photovoltages and photocurrents (Figure 4d and Figure S16, Supporting Information). ${ }^{41,42}$ When the $\mathrm{FeOOH} / 1 \mathrm{~L} / 3 \mathrm{C}-\mathrm{SiC}$ photoanode was used with the $\mathrm{Pt}$ as the counter electrode, dominant reduction product was $\mathrm{H}_{2}(91.2 \%)$ with $\mathrm{CO}(7.5 \%)$ as the byproduct, suggesting that the tuned graphene/3C-SiC photoanode can also be used for solar-to-hydrogen conversion. The selectivity of the $\mathrm{CO}_{2}$ reduction on different metal anodes has been well-studied. ${ }^{43}$ This work, however, focuses on the atomic tuning of the graphene/3C-SiC Schottky junction for promoting the charge separation and transport. As a proof-ofconcept, such an atomically tuned junction shows a promising PEC performance for solar-to-fuel conversion. We further measured potential between the working electrode $\left(E_{\mathrm{WE}}\right)$ and counter electrode $\left(E_{\mathrm{CE}}\right)\left(\left|E_{\mathrm{WE}}-E_{\mathrm{CE}}\right|\right)$ during $\mathrm{CO}_{2}$ reduction. Under a bias of $+0.6 \mathrm{~V}_{\mathrm{RHE}}, \mathrm{FeOOH} / 1 \mathrm{~L} / 3 \mathrm{C}-\mathrm{SiC}$ photoanodedriven $\mathrm{PEC}$ with $\mathrm{Cu}$ as the cathode has a $\left|E_{\mathrm{WE}}-E_{\mathrm{CE}}\right|$ of 1.43 $\mathrm{V}$. For comparison, in the $\mathrm{PEC} \mathrm{CO}_{2}$ reduction system using $\mathrm{CuO}_{x}$ as the photocathode and $\mathrm{Pt}$ as the anode, the $\mid E_{\mathrm{WE}}-$ $E_{\mathrm{CE}} \mid$ was reported to be around $1.80 \mathrm{~V}$ at a low potential of $+0.07 \mathrm{~V}_{\mathrm{RHE}}$. Therefore, photoanode-driven $\mathrm{CO}_{2}$ reduction based on the $\mathrm{FeOOH} / 1 \mathrm{~L} / 3 \mathrm{C}$ - $\mathrm{SiC}$ photoanode is very promising as it requires less external energy input. ${ }^{41,44}$ When isotopic ${ }^{13} \mathrm{CO}_{2}$ was used as the carbon source, ${ }^{13} \mathrm{CH}_{4}$ with a mass/charge ratio $(\mathrm{m} / z)$ of 17 was detected as the only product in the $\mathrm{FeOOH} / 1 \mathrm{~L} / 3 \mathrm{C}$-SiC photoanodellCu cathode PEC system (Figure 4e). Meanwhile, the identical abundance of the corresponding fragments of ${ }^{13} \mathrm{CH}_{4}$ and ${ }^{12} \mathrm{CH}_{4}$ further indicated that photocorrosion of $3 \mathrm{C}-\mathrm{SiC}$ was suppressed by protective graphene layers. ${ }^{45,46} \mathrm{In}$ addition to the $\mathrm{Cu}$ cathode, when a commercial $\mathrm{Zn}$ cathode or a $\mathrm{Bi}$ cathode was coupled with the $\mathrm{FeOOH} / 1 \mathrm{~L} / 3 \mathrm{C}$-SiC photoanode, $\mathrm{CO}$ and formic acid emerged as the respective selective $\mathrm{CO}_{2}$ reduction products (Figure $4 \mathrm{f}$ and Table S2, Supporting Information). This proof-of-concept PEC cell revealed the versatility of $\mathrm{FeOOH} / 1 \mathrm{~L} / 3 \mathrm{C}-\mathrm{SiC}$ as the efficient photoanode to drive a selective conversion of $\mathrm{CO}_{2}$ into desirable solar fuels.

\section{CONCLUSIONS}

In conclusion, we present an interesting atomic Schottky junction between epitaxial graphene layers and 3C-SiC substrates. By tailoring the number of graphene layers, this junction structure exhibits an atomic-scale-tunable Schottky junction with an inherent built-in electric field distributed across up to the fourth graphene layer. As a proof-of-concept study, the atomic-scale-tuned Schottky junction is demonstrated to promote both the separation and transport of charge carriers under solar light, thus enhancing the overall photoelectrochemical performance of the solar-to-fuel conversion, including increased photocurrent and photovoltage together with a reduced onset potential. Simultaneously, the as-grown monolayer graphene with an extremely high conductivity protects the surface of $3 \mathrm{C}-\mathrm{SiC}$ from photocorrosion and energetically delivers charge carriers to the loaded cocatalyst, resulting in a synergetic enhancement of both the stability and the efficiency of this system. The knowledge gained in this study not only highlights the paramount importance of sophisticated interfacial structure design for construction of feasible renewable energy systems but also illuminates the engineering of graphene-based devices.

\section{METHODS}

Preparation of the $3 \mathrm{C}$-SiC Substrate. Through a sublimation process, $3 \mathrm{C}-\mathrm{SiC}(111)$ with a thickness around $1 \mathrm{~mm}$ was grown on a $4^{\circ}$ off-axis n-type $4 \mathrm{H}-\mathrm{SiC}$ (SiCrystal). ${ }^{38,47}$ To obtain single-crystal 3C$\mathrm{SiC}$ with a thickness around $300 \mu \mathrm{m}$, the $4 \mathrm{H}-\mathrm{SiC}$ substrate and transition interface between the two polytypes were carefully polished 
away. After that, the $3 \mathrm{C}-\mathrm{SiC}(111)$ substrate was thoroughly cleaned with acetone, ethanol, $\mathrm{H}_{2} \mathrm{O} / \mathrm{NH}_{3} / \mathrm{H}_{2} \mathrm{O}_{2}$ (5:1:1), $\mathrm{H}_{2} \mathrm{O} / \mathrm{HCl} / \mathrm{H}_{2} \mathrm{O}_{2}$ (6:1:1), and hydrofluoric acid. Prior to graphene growth, the obtained clean 3C-SiC(111) substrate was checked by optical microscopy and atomic force microscopy to ensure there were no macroscopic defects. Doping concentration $\left(N_{\mathrm{D}}\right)$ of the n-type $3 \mathrm{C}$-SiC was measured to be around $7.5 \times 10^{15} \mathrm{~cm}^{-3}$.

Preparation of the Graphene/3C-SiC Junction. An inductively heated furnace was used to grow graphene on the $3 \mathrm{C}-\mathrm{SiC}$ substrate under $1800{ }^{\circ} \mathrm{C}$ in an $850 \mathrm{mbar}$ argon atmosphere for different times. ${ }^{16}$ The ramping rate was set at $25{ }^{\circ} \mathrm{C} / \mathrm{min}$. For the preparation of $\mathrm{BL} / 3 \mathrm{C}-\mathrm{SiC}, 1 \mathrm{~L} / 3 \mathrm{C}-\mathrm{SiC}$, and $2 \mathrm{~L} / 3 \mathrm{C}-\mathrm{SiC}$, the $3 \mathrm{C}-\mathrm{SiC}$ was annealed at $1800{ }^{\circ} \mathrm{C}$ for 1,15 , and $30 \mathrm{~min}$, respectively. The thicker graphene of more than two layers can be grown by tuning the growth temperature and pressure. As it was very challenging to grow thick $(>3 \mathrm{~L})$ graphene on the $\mathrm{Si}$-face $\mathrm{SiC}$ substrate, we focused on how to grow graphene with four layers. However, we believe the effect of $1 \mathrm{~L}$, $2 \mathrm{~L}$, and $4 \mathrm{~L}$ graphene on charge transfer as we discussed above should be applicable to the $3 \mathrm{~L}$ graphene. In this work, four-layer graphene, as an example, was grown at a higher temperature of $2000^{\circ} \mathrm{C}$ in an 850 mbar argon atmosphere for $30 \mathrm{~min}$. For $\mathrm{FeOOH}$ nanorod deposition, graphene/3C-SiC samples were immersed in a precursor solution containing $30 \mathrm{mM} \mathrm{FeCl}_{3}$ and $45 \mathrm{mM}$ urea. ${ }^{48}$ The urea was used as the progressive $\mathrm{OH}^{-}$releasing agent. The above mixture was then kept for $30 \mathrm{~min}$ at $100{ }^{\circ} \mathrm{C}$ to enable rapid $\mathrm{FeOOH}$ deposition. After the deposition, the coated photoanodes were rinsed with DI water and then nitrogen-dried. Sheet resistance of the used FTO glass to support the $\mathrm{Cu}$ tape and graphene/3C-SiC samples is around $8 \mathrm{ohms}$ per square. For $\mathrm{CoOOH}$ nanoparticle deposition, $3 \mathrm{C}-\mathrm{SiC}$ was used as the working electrode, $\mathrm{Ag} / \mathrm{AgCl}$ as the reference electrode, and $\mathrm{Pt}$ mesh as the counter electrode in a typical three-electrode cell. Next, 0.6 $\mathrm{V}_{\mathrm{RHE}}$ was applied in $0.01 \mathrm{~mol} / \mathrm{L} \mathrm{CoCl}_{2}$ aqueous solution under simulated solar light irradiation (AM1.5G, $100 \mathrm{~mW} / \mathrm{cm}^{2}$ ) with a deposition time of $30 \mathrm{~min}^{49}$ Because the $1 \mathrm{~L} / 3 \mathrm{C}-\mathrm{SiC}$ photoanode possessed a photocurrent density much higher than that of the 3C$\mathrm{SiC}$ photoanode under the same bias, the $\mathrm{CoOOH}$ deposition time was shortened accordingly to ensure the same accumulated charge passing through the photoanodes. This could ensure the comparable amount of $\mathrm{CoOOH}$ deposited on the $1 \mathrm{~L} / 3 \mathrm{C}-\mathrm{SiC}$ photoanode.

DFT Theoretical Calculations. Theoretical calculations were performed using DFT with the exchange-correlation energy functional implemented in the Vienna $A b$ initio Simulation Package, as described by the generalized gradient approximation with the Perdew-BurkeErnzerhof exchange-correlation function. ${ }^{50}$ To describe electron-ion interactions, the projector-augmented wave method was applied with a plane-wave cutoff energy of $400 \mathrm{eV}^{50,51}$ For geometric optimizations and DOS calculations, the energy and force converged to $10^{-5} \mathrm{eV}$ atom ${ }^{-1}$ and $0.02 \mathrm{eV} \AA^{-1}$, and the corresponding K-point was $3 \times 2 \times 1$. Si-terminated $3 \mathrm{C}$-SiC modified with graphene heterojunctions was established according to the suitable lattice match, evidenced by $30^{\circ}$ offset in two lattice hexagons. Multilayer graphene was added on the basis of graphene growth. The simulative supercells $(3 \times 3 \times 1)$ with a vacuum thickness of $20 \AA$ were denoted as $\mathrm{Si}$-terminated $3 \mathrm{C}$-SiC modified with graphene heterojunctions. The charge density difference was calculated as $\Delta \rho=\rho$ (graphene/3C-SiC) $-\rho(3 \mathrm{C}-\mathrm{SiC})-\rho$ (graphene). The $\rho$ (graphene/3C-SiC) was denoted as the density of heterojunctions, whereas $\rho(3 \mathrm{C}-\mathrm{SiC})$ and $\rho$ (graphene) were denoted as the densities of the two subsystems. The electrostatic potential and work function calculation were performed with CASTEP, in which the plane-wave pseudopotential with an energy cutoff of $310 \mathrm{eV}$ was employed for all calculation systems, and the corresponding energy and force convergence were $10^{-5} \mathrm{eV}$ atom ${ }^{-1}$ and $0.02 \mathrm{eV} \AA^{-1}$, respectively.

Characterization of Graphene/3C-SiC Junctions. Atomic graphene layers were first determined by cross-sectional HRTEM (FEI Tecnai G2 TF 20 UT) operated at $200 \mathrm{keV}$. The number of multiple graphene layers, quality, and the uniformity of the graphene layers were determined by LEEM and $\mu$-LEED in ultrahigh vacuum, which were carried out using a SPELEEM instrument at beamline I311 in the MAX IV Laboratory synchrotron radiation laboratory,
Lund, Sweden. The $\mu$-LEED patterns were obtained from the selected areas (range: $500-1000 \mathrm{~nm}$ ), matching the size of the corresponding graphene domain. Room temperature ARPES data were recorded in an ultrahigh vacuum system at Linköping University, Sweden, using a Phoibos 100 analyzer from Specs, equipped with a two-dimensional detector and HeI radiation $(h \nu=21.22 \mathrm{eV})$ from a nonmonochromatized resonance lamp. Energy and angular resolutions were $50 \mathrm{meV}$ and $0.3^{\circ}$, respectively. The position of the Fermi level was determined using spectra recorded from a clean $\mathrm{Ta}$ foil in electrical contact with the samples. Work function data were derived from the position of the cutoff of the secondary electron emission. A negative bias around $-9.526 \mathrm{~V}$ was applied to the samples relative to ground. This results in an increase of the kinetic energy of the emitted electrons, which is necessary in order to obtain well-defined and steep edges in the cutoff spectra. Surface morphology was determined by scanning electron microscopy (LEO 1550 Gemini, $10 \mathrm{kV}$ ). XRD measurements were conducted using a Philips MRD with $\mathrm{Cu} \mathrm{K} \alpha 1$ ( $\lambda$ $=1.54 \AA$ ).

Photoelectrochemical Water Oxidation and $\mathrm{CO}_{2}$ Reduction. The designed photoelectrochemical reactor was made of a photoanode and a cathode compartment, which were separated by a Nafion-115 proton exchange membrane. We used $0.5 \mathrm{M}$ pH 7.5 $\mathrm{KHCO}_{3}$ solution as the electrolyte, and the photoanode compartment was bubbled by high-purity $\mathrm{Ar}$ and cathode compartment by highpurity $\mathrm{CO}_{2}$. Graphene/3C-SiC, selected metal, and $\mathrm{Ag} / \mathrm{AgCl}$ were used, respectively, as the photoanode, cathode, and reference electrode. The photoanode and cathode compartment were connected to a gas circulation system for online sampling and analysis through a gas chromatograph system (GC: Agilent HP 6890). The GC system has a Carboxen-1000 packing column to separate and analyze gaseous mixtures of $\mathrm{O}_{2}, \mathrm{CO}, \mathrm{CH}_{4}, \mathrm{C}_{2} \mathrm{H}_{4}$, and $\mathrm{H}_{2}$. Liquid product of formic acid was determined by an ionic chromatograph (Thermo Scientific Dionex, ICS-900). Both the electrolyte and the circulation system were purged with $\mathrm{CO}_{2}$ for $60 \mathrm{~min}$ before the photoelectrochemical catalysis. Particularly, in the cathode compartment, electrolyte was pre-electrolyzed at $0.025 \mathrm{~mA}$ using $\mathrm{Pt}$ mesh for 120 min under $\mathrm{CO}_{2}$ purging to remove the metal ion impurities and to avoid possible cathode poisoning. The whole photoelectrochemical process was conducted under atmospheric pressure of $\mathrm{CO}_{2}$. The bias was applied using a using a potentiostat/EIS (Princeton Applied Research, VersaSTAT 3). All potentials in this work are with respect to $\mathrm{Ag} / \mathrm{AgCl}$ and converted to potentials versus reversible hydrogen electrode (RHE) according to the equation: $V_{\mathrm{RHE}}=V_{\mathrm{Ag} / \mathrm{AgCl}}+0.197+$ $0.059 \times \mathrm{pH}$. Faradaic efficiencies for the photoelectrochemical reactions were calculated based on the utilized charge and the number of holes or electrons required for the formation of certain products. For an isotopic labeling experiment, ${ }^{13} \mathrm{CH}_{4}$ was collected and analyzed via a gas chromatograph-mass spectrometer (Pfeiffer OmniStar) using ${ }^{13} \mathrm{CO}_{2}\left({ }^{13} \mathrm{C} 99 \%\right.$, Sigma-Aldrich) as the carbon source. For open-circuit potential measurements, $\mathrm{O}_{2}$ gas was continuously bubbled into the electrolyte $\left(0.5 \mathrm{M} \mathrm{pH} 7.5 \mathrm{KHCO}_{3}\right)$, and each dark/light open-circuit potential was obtained with constant stirring.

\section{ASSOCIATED CONTENT}

\section{Supporting Information}

The Supporting Information is available free of charge at https://pubs.acs.org/doi/10.1021/acsnano.0c00986.

Raman spectra of the synthesized materials, DOS of BL/ $3 \mathrm{C}-\mathrm{SiC}$, energy band diagram for graphene/3C-SiC junction, UPS of the synthesized materials, calculated electrostatic potential energy, EIS plots, characterization of $\mathrm{FeOOH} / 1 \mathrm{~L} / 3 \mathrm{C}-\mathrm{SiC}$ and $\mathrm{CoOOH} / 1 \mathrm{~L} / 3 \mathrm{C}-\mathrm{SiC}$, supporting figures and tables (PDF) 


\section{AUTHOR INFORMATION}

\section{Corresponding Author}

Jianwu Sun - Department of Physics, Chemistry and Biology (IFM), Linköping University, 58183 Linköping, Sweden; ○ orcid.org/0000-0002-6403-3720; Email: jianwu.sun@ liu.se

\section{Authors}

Hao Li - Department of Physics, Chemistry and Biology (IFM), Linköping University, 58183 Linköping, Sweden; 이이이.org/ 0000-0002-1135-8353

Yuchen Shi - Department of Physics, Chemistry and Biology (IFM), Linköping University, 58183 Linköping, Sweden; () orcid.org/0000-0002-8904-9884

Huan Shang - Key Laboratory of Pesticide \& Chemical Biology of Ministry of Education, Institute of Applied \& Environmental Chemistry, College of Chemistry, Central China Normal University, Wuhan 430079, P.R. China

Weimin Wang - Department of Physics, Chemistry and Biology (IFM), Linköping University, 58183 Linköping, Sweden; MAX IV Laboratory, SE-22484 Lund, Sweden

Jun Lu - Department of Physics, Chemistry and Biology (IFM), Linköping University, 58183 Linköping, Sweden

Alexei A. Zakharov - MAX IV Laboratory, SE-22484 Lund, Sweden; (1) orcid.org/0000-0002-1269-6813

Lars Hultman - Department of Physics, Chemistry and Biology (IFM), Linköping University, 58183 Linköping, Sweden

Roger I. G. Uhrberg - Department of Physics, Chemistry and Biology (IFM), Linköping University, 58183 Linköping, Sweden

Mikael Syväjärvi - Department of Physics, Chemistry and Biology (IFM), Linköping University, 58183 Linköping, Sweden

Rositsa Yakimova - Department of Physics, Chemistry and Biology (IFM), Linköping University, 58183 Linköping, Sweden

Lizhi Zhang - Key Laboratory of Pesticide \& Chemical Biology of Ministry of Education, Institute of Applied \& Environmental Chemistry, College of Chemistry, Central China Normal University, Wuhan 430079, P.R. China; (1) orcid.org/00000002-6842-9167

Complete contact information is available at:

https://pubs.acs.org/10.1021/acsnano.0c00986

\section{Notes}

The authors declare no competing financial interest.

\section{ACKNOWLEDGMENTS}

This work was supported by The Swedish Research Council (Vetenskapsrådet, Grant Nos. 621-2014-5461, 2018-04670, 621-2014-5805, and 2018-04962), The Swedish Research Council for Environment, Agricultural Sciences and Spatial Planning (FORMAS, Grant No. 2016-00559), The Swedish Foundation for International Cooperation in Research and Higher Education (STINT, Grant No. CH2016-6722), The ÅForsk foundation (Grant Nos. 16-399 and 18-370), and The Stiftelsen Olle Engkvist Byggmästare (Grant No. 189-0243). A.A.Z. and R.Y. would like to acknowledge support from Siftelsen för Strategisk Forskning (Project RMA15-0024).

\section{REFERENCES}

(1) Allen, M. J.; Tung, V. C.; Kaner, R. B. Honeycomb Carbon: A Review of Graphene. Chem. Rev. 2010, 110, 132-145.

(2) Sun, Y.; Wu, Q.; Shi, G. Graphene Based New Energy Materials. Energy Environ. Sci. 2011, 4, 1113.
(3) Chang, H.; Wu, H. Graphene-Based Nanocomposites: Preparation, Functionalization, and Energy and Environmental Applications. Energy Environ. Sci. 2013, 6, 3483-3507.

(4) Du, A.; Sanvito, S.; Li, Z.; Wang, D.; Jiao, Y.; Liao, T.; Sun, Q.; Ng, Y. H.; Zhu, Z.; Amal, R.; Smith, S. Hybrid Graphene and Graphitic Carbon Nitride Nanocomposite: Gap Opening, ElectronHole Puddle, Interfacial Charge Transfer, and Enhanced Visible Light Response. J. Am. Chem. Soc. 2012, 134, 4393-4397.

(5) Eda, G.; Lin, Y.-Y.; Mattevi, C.; Yamaguchi, H.; Chen, H.-A.; Chen, I.-S.; Chen, C.-W.; Chhowalla, M. Blue Photoluminescence from Chemically Derived Graphene Oxide. Adv. Mater. 2010, 22, 505-509.

(6) Novoselov, K. S. Electric Field Effect in Atomically Thin Carbon Films. Science 2004, 306, 666-669.

(7) Di Bartolomeo, A. Graphene Schottky Diodes: An Experimental Review of the Rectifying Graphene/Semiconductor Heterojunction. Phys. Rep. 2016, 606, 1-58.

(8) Wang, Z.-J.; Dong, J.; Cui, Y.; Eres, G.; Timpe, O.; Fu, Q.; Ding, F.; Schloegl, R.; Willinger, M.-G. Stacking Sequence and Interlayer Coupling in Few-Layer Graphene Revealed by In Situ Imaging. Nat. Commun. 2016, 7, 13256.

(9) Hass, J.; Varchon, F.; Millán-Otoya, J. E.; Sprinkle, M.; Sharma, N.; de Heer, W. A.; Berger, C.; First, P. N.; Magaud, L.; Conrad, E. H. Why Multilayer Graphene on $4 \mathrm{H}-\mathrm{SiC}(000-1)$ Behaves Like a Single Sheet of Graphene. Phys. Rev. Lett. 2008, 100, 125504.

(10) Lui, C. H.; Li, Z.; Mak, K. F.; Cappelluti, E.; Heinz, T. F. Observation of an Electrically Tunable Band Gap in Trilayer Graphene. Nat. Phys. 2011, 7, 944-947.

(11) Coletti, C.; Forti, S.; Principi, A.; Emtsev, K. V.; Zakharov, A. A.; Daniels, K. M.; Daas, B. K.; Chandrashekhar, M. V. S.; Ouisse, T.; Chaussende, D.; MacDonald, A. H.; Polini, M.; Starke, U. Revealing the Electronic Band Structure of Trilayer Graphene on SiC: An Angle-Resolved Photoemission Study. Phys. Rev. B: Condens. Matter Mater. Phys. 2013, 88, 155439.

(12) Zhang, Y.; Tang, T.-T.; Girit, C.; Hao, Z.; Martin, M. C.; Zettl, A.; Crommie, M. F.; Shen, Y. R.; Wang, F. Direct Observation of a Widely Tunable Bandgap in Bilayer Graphene. Nature 2009, 459, 820-823.

(13) Kuang, Y.; Jia, Q.; Ma, G.; Hisatomi, T.; Minegishi, T.; Nishiyama, H.; Nakabayashi, M.; Shibata, N.; Yamada, T.; Kudo, A.; Domen, K. Ultrastable Low-Bias Water Splitting Photoanodes via Photocorrosion Inhibition and In Situ Catalyst Regeneration. Nat. Energy 2017, 2, 16191.

(14) Sokol, K. P.; Robinson, W. E.; Warnan, J.; Kornienko, N.; Nowaczyk, M. M.; Ruff, A.; Zhang, J. Z.; Reisner, E. Bias-Free Photoelectrochemical Water Splitting with Photosystem II on a DyeSensitized Photoanode Wired to Hydrogenase. Nat. Energy 2018, 3, 944-951.

(15) Yu, Y.; Zhang, Z.; Yin, X.; Kvit, A.; Liao, Q.; Kang, Z.; Yan, X.; Zhang, Y.; Wang, X. Enhanced Photoelectrochemical Efficiency and Stability Using a Conformal $\mathrm{TiO}_{2}$ Film on a Black Silicon Photoanode. Nat. Energy 2017, 2, 17045.

(16) Shi, Y.; Zakharov, A. A.; Ivanov, I. G.; Yazdi, G. R.; Jokubavicius, V.; Syväjärvi, M.; Yakimova, R.; Sun, J. Elimination of Step Bunching in the Growth of Large-Area Monolayer and Multilayer Graphene on Off-Axis 3C SiC (111). Carbon 2018, 140, 533-542.

(17) Van Bommel, A. J.; Crombeen, J. E.; Van Tooren, A. LEED and Auger Electron Observations of the $\mathrm{SiC}(0001)$ Surface. Surf. Sci. 1975, 48, 463-472.

(18) Riedl, C.; Starke, U.; Bernhardt, J.; Franke, M.; Heinz, K. Structural Properties of the Graphene-SiC(0001) Interface as a Key for the Preparation of Homogeneous Large-Terrace Graphene Surfaces. Phys. Rev. B: Condens. Matter Mater. Phys. 2007, 76, 245406.

(19) Emtsev, K. V.; Speck, F.; Seyller, T.; Ley, L.; Riley, J. D. Interaction, Growth, and Ordering of Epitaxial Graphene on SiC $\{0001\}$ Surfaces: A Comparative Photoelectron Spectroscopy Study. Phys. Rev. B: Condens. Matter Mater. Phys. 2008, 77, 155303. 
(20) Wang, W.; Shi, Y.; Zakharov, A. A.; Syväjärvi, M.; Yakimova, R.; Uhrberg, R. I. G.; Sun, J. Flat-Band Electronic Structure and Interlayer Spacing Influence in Rhombohedral Four-Layer Graphene. Nano Lett. 2018, 18, 5862-5866.

(21) Mattausch, A.; Pankratov, O. Ab Initio Study of Graphene on SiC. Phys. Rev. Lett. 2007, 99, 076802.

(22) Riedl, C.; Coletti, C.; Iwasaki, T.; Zakharov, A. A.; Starke, U. Quasi-Free-Standing Epitaxial Graphene on $\mathrm{SiC}$ Obtained by Hydrogen Intercalation. Phys. Rev. Lett. 2009, 103, 246804.

(23) Borysiuk, J.; Soltys, J.; Piechota, J. Stacking Sequence Dependence of Graphene Layers on SiC (0001-) -Experimental and Theoretical Investigation. J. Appl. Phys. 2011, 109, 093523.

(24) Hibino, H.; Kageshima, H.; Maeda, F.; Nagase, M.; Kobayashi, Y.; Yamaguchi, H. Microscopic Thickness Determination of Thin Graphite Films Formed on $\mathrm{SiC}$ from Quantized Oscillation in Reflectivity of Low-Energy Electrons. Phys. Rev. B: Condens. Matter Mater. Phys. 2008, 77, 075413.

(25) Ni, Z. H.; Chen, W.; Fan, X. F.; Kuo, J. L.; Yu, T.; Wee, A. T. S.; Shen, Z. X. Raman Spectroscopy of Epitaxial Graphene on a SiC Substrate. Phys. Rev. B: Condens. Matter Mater. Phys. 2008, 77, 115416.

(26) Long, R.; English, N. J.; Prezhdo, O. V. Photo-Induced Charge Separation across the Graphene- $\mathrm{TiO}_{2}$ Interface Is Faster than Energy Losses: A Time-Domain Ab Initio Analysis. J. Am. Chem. Soc. 2012, 134, 14238-14248.

(27) Bardeen, J. Surface States and Rectification at a Metal SemiConductor Contact. Phys. Rev. 1947, 71, 717-727.

(28) Mammadov, S.; Ristein, J.; Krone, J.; Raidel, C.; Wanke, M.; Wiesmann, V.; Speck, F.; Seyller, T. Work Function of Graphene Multilayers on $\mathrm{SiC}(0001)$. 2D Mater. 2017, 4, 015043.

(29) Bai, S.; Wang, C.; Deng, M.; Gong, M.; Bai, Y.; Jiang, J.; Xiong, Y. Surface Polarization Matters: Enhancing the Hydrogen-Evolution Reaction by Shrinking Pt Shells in Pt-Pd-Graphene Stack Structures. Angew. Chem., Int. Ed. 2014, 53, 12120-12124.

(30) Riedl, C.; Coletti, C.; Starke, U. Structural and Electronic Properties of Epitaxial Graphene on $\operatorname{SiC}\left(\begin{array}{llll}0 & 0 & 0 & 1\end{array}\right)$ : A Review of Growth, Characterization, Transfer Doping and Hydrogen Intercalation. J. Phys. D: Appl. Phys. 2010, 43, 374009.

(31) Lounis, S. D.; Siegel, D. A.; Broesler, R.; Hwang, C. G.; Haller, E. E.; Lanzara, A. Resonant Photoluminescent Charging of Epitaxial Graphene. Appl. Phys. Lett. 2010, 96, 151913.

(32) Pierucci, D.; Sediri, H.; Hajlaoui, M.; Girard, J.-C.; Brumme, T.; Calandra, M.; Velez-Fort, E.; Patriarche, G.; Silly, M. G.; Ferro, G.; Soulière, V.; Marangolo, M.; Sirotti, F.; Mauri, F.; Ouerghi, A. Evidence for Flat Bands Near the Fermi Level in Epitaxial Rhombohedral Multilayer Graphene. ACS Nano 2015, 9, 5432-5439.

(33) Davydov, S. Y. On the Electron Affinity of Silicon Carbide Polytypes. Semiconductors 2007, 41, 696-698.

(34) Li, H.; Shang, H.; Shi, Y.; Yakimova, R.; Syväjärvi, M.; Zhang, L.; Sun, J. Atomically Manipulated Proton Transfer Energizes Water Oxidation on Silicon Carbide Photoanodes. J. Mater. Chem. A 2018, 6, 24358-24366.

(35) Arai, T.; Sato, S.; Kajino, T.; Morikawa, T. Solar $\mathrm{CO}_{2}$ Reduction Using $\mathrm{H}_{2} \mathrm{O}$ by a Semiconductor/Metal-Complex Hybrid Photocatalyst: Enhanced Efficiency and Demonstration of a Wireless System Using $\mathrm{SrTiO}_{3}$ Photoanodes. Energy Environ. Sci. 2013, 6, $1274-1282$.

(36) Kim, J. Y.; Youn, D. H.; Kang, K.; Lee, J. S. Highly Conformal Deposition of an Ultrathin $\mathrm{FeOOH}$ Layer on a Hematite Nanostructure for Efficient Solar Water Splitting. Angew. Chem., Int. Ed. 2016, 55, 10854-10858.

(37) Du, C.; Yang, X.; Mayer, M. T.; Hoyt, H.; Xie, J.; McMahon, G.; Bischoping, G.; Wang, D. Hematite-Based Water Splitting with Low Turn-On Voltages. Angew. Chem., Int. Ed. 2013, 52, 1269212695 .

(38) Jokubavicius, V.; Yazdi, G. R.; Liljedahl, R.; Ivanov, I. G.; Yakimova, R.; Syväjärvi, M. Lateral Enlargement Growth Mechanism of 3C-SiC on Off-Oriented $4 \mathrm{H}-\mathrm{SiC}$ Substrates. Cryst. Growth Des. 2014, 14, 6514-6520.
(39) van Dorp, D. H.; Kelly, J. J. Photoelectrochemistry of 4H-SiC in KOH Solutions. J. Electroanal. Chem. 2007, 599, 260-266.

(40) Nielander, A. C.; Bierman, M. J.; Petrone, N.; Strandwitz, N. C.; Ardo, S.; Yang, F.; Hone, J.; Lewis, N. S. Photoelectrochemical Behavior of $n$-Type Si(111) Electrodes Coated With a Single Layer of Graphene. J. Am. Chem. Soc. 2013, 135, 17246-17249.

(41) Kim, J. H.; Magesh, G.; Kang, H. J.; Banu, M.; Kim, J. H.; Lee, J.; Lee, J. S. Carbonate-Coordinated Cobalt Co-Catalyzed $\mathrm{BiVO}_{4} /$ $\mathrm{WO}_{3}$ Composite Photoanode Tailored for $\mathrm{CO}_{2}$ Reduction to Fuels. Nano Energy 2015, 15, 153-163.

(42) Magesh, G.; Kim, E. S.; Kang, H. J.; Banu, M.; Kim, J. Y.; Kim, J. H.; Lee, J. S. A Versatile Photoanode-Driven Photoelectrochemical System for Conversion of $\mathrm{CO}_{2}$ to Fuels With High Faradaic Efficiencies at Low Bias Potentials. J. Mater. Chem. A 2014, 2, 2044-2049.

(43) Nitopi, S.; Bertheussen, E.; Scott, S. B.; Liu, X.; Engstfeld, A. K.; Horch, S.; Seger, B.; Stephens, I. E. L.; Chan, K.; Hahn, C.; Nørskov, J. K.; Jaramillo, T. F.; Chorkendorff, I. Progress and Perspectives of Electrochemical $\mathrm{CO}_{2}$ Reduction on Copper in Aqueous Electrolyte. Chem. Rev. 2019, 119, 7610-7672.

(44) Ghadimkhani, G.; de Tacconi, N. R.; Chanmanee, W.; Janaky, C.; Rajeshwar, K. Efficient Solar Photoelectrosynthesis of Methanol from Carbon Dioxide Using Hybrid $\mathrm{CuO}-\mathrm{Cu}_{2} \mathrm{O}$ Semiconductor Nanorod Arrays. Chem. Commun. 2013, 49, 1297.

(45) Sekimoto, T.; Hashiba, H.; Shinagawa, S.; Uetake, Y.; Deguchi, M.; Yotsuhashi, S.; Ohkawa, K. Analysis of Products from Photoelectrochemical Reduction of ${ }^{13} \mathrm{CO}_{2}$ by GaN-Si Based Tandem Photoelectrode. J. Phys. Chem. C 2016, 120, 13970-13975.

(46) Yu, L.; Li, G.; Zhang, X.; Ba, X.; Shi, G.; Li, Y.; Wong, P. K.; Yu, J. C.; Yu, Y. Enhanced Activity and Stability of Carbon-Decorated Cuprous Oxide Mesoporous Nanorods for $\mathrm{CO}_{2}$ Reduction in Artificial Photosynthesis. ACS Catal. 2016, 6, 6444-6454.

(47) Jokubavicius, V.; Yazdi, G. R.; Liljedahl, R.; Ivanov, I. G.; Sun, J.; Liu, X.; Schuh, P.; Wilhelm, M.; Wellmann, P.; Yakimova, R.; Syväjärvi, M. Single Domain 3C-SiC Growth on Off-Oriented 4H-SiC Substrates. Cryst. Growth Des. 2015, 15, 2940-2947.

(48) Cai, L.; Zhao, J.; Li, H.; Park, J.; Cho, I. S.; Han, H. S.; Zheng, X. One-Step Hydrothermal Deposition of Ni:FeOOH onto Photoanodes for Enhanced Water Oxidation. ACS Energy Lett. 2016, 1, 624-632.

(49) Xu, Z.; Li, X.; Li, J.; Wu, L.; Zeng, Q.; Zhou, Z. Effect of $\mathrm{CoOOH}$ Loading on the Photoelectrocatalytic Performance of $\mathrm{WO}_{3}$ Nanorod Array Film. Appl. Surf. Sci. 2013, 284, 285-290.

(50) Perdew, J. P.; Burke, K.; Ernzerhof, M. Generalized Gradient Approximation Made Simple. Phys. Rev. Lett. 1996, 77, 3865-3868.

(51) Vanderbilt, D. Soft Self-Consistent Pseudopotentials in a Generalized Eigenvalue Formalism. Phys. Rev. B: Condens. Matter Mater. Phys. 1990, 41, 7892-7895. 